\title{
A RIGIDITY THEOREM FOR DISCRETE SUBGROUPS
}

\author{
BY \\ HOWARD GARLAND
}

0. Introduction. Let $G$ denote an analytic group and let $\Gamma \subset G$ denote a discrete subgroup. A left invariant Haar measure on $G$ then induces a measure on $G / \Gamma$, which is determined up to a positive constant. We let $v$ denote one such induced measure on $G / \Gamma$.

Definition 0.1. $\Gamma$ is called a lattice, in case $v(G / \Gamma)<\infty$.

Unless specified otherwise, $\Gamma$ will denote a lattice in the analytic group $G$. Let $W$ be a simply connected $C^{\infty}$ manifold; let $\mathscr{R}$ denote the set of all one-one homomorphisms

$$
r: \Gamma \rightarrow G
$$

such that $r(\Gamma)$ is a lattice in $G$. We say a map $\chi: W \rightarrow \mathscr{R}$ is smooth if for all $\gamma \in \Gamma$, the map $w \rightarrow \chi(w)(\gamma)$, (where $w \in W$ ), is a $C^{\infty}$ map of $W$ into $G$.

Definition 0.2. A deformation of $\Gamma$ is the data $\left(W, w_{0}, \chi\right)$, where $W$ is a simply connected $C^{\infty}$ manifold, $w_{0}$ is a point in $W$, and $\chi: W \rightarrow R$ is a smooth map such that $\chi\left(w_{0}\right)$ is the inclusion map $\iota$, of $\Gamma$ into $G$.

Definition 0.3. The deformation $\left(W, w_{0}, \chi\right)$ is called trivial, in case we can find a $C^{\infty} \operatorname{map} c: W \rightarrow G$, such that for all $w \in W$ and for all $\gamma \in \Gamma$, we have

$$
\chi(w)(\gamma)=c(w) \gamma c(w)^{-1}
$$

We say $\Gamma$ is rigid if all its deformations are trivial. We say that $G$ is admissible, in case it is semisimple and has no compact or three dimensional factors. We make the convention that the Lie algebra of a Lie group is the tangent space of that group at the identity. An analytic subgroup and its corresponding Lie subalgebra will be denoted by the same capital Latin and capital German letter, respectively. Thus if $G$ is an analytic group and $A \subset G$ an analytic subgroup, (Bs will denote the Lie algebra of $G$, and $\mathscr{U} \subset \mathbb{B S}$ will denote the subalgebra corresponding to $A$. Whenever $\boldsymbol{G}$ is admissible, we fix a Cartan decomposition $\mathbb{S}=\mathfrak{\Omega}+\mathfrak{P}$, and we let $\boldsymbol{M}=K \mid \boldsymbol{G}$.

In this paper we will be interested in the following:

Conjecture (A. Selberg). Let $G$ be admissible and let $\Gamma \subset G$ be a lattice; then all deformations of $\Gamma$ are trivial.

To attack this conjecture, we adopt the point of view in [13]. Thus let $\omega^{1}, \ldots, \omega^{n}$ (where $n=\operatorname{dimension} G$ ) be a basis of right-invariant one-forms on $G$. By the rightinvariance of the $\omega^{\lambda}(\lambda=1, \ldots, n)$ we have everywhere independent, induced forms

Received by the editors September 6, 1966. 
on $G / \Gamma$, which we again denote by $\omega^{\lambda}$. Thus we have a parallelism structure on $G / \Gamma$. Roughly speaking, we may think of a deformation $\left(W, w_{0}, \chi\right)$ of $\Gamma$ as inducing a deformation of this parallelism structure. We show that if $G$ is admissible, if $\Gamma$ contains no nontrivial elements of finite $\operatorname{order}\left({ }^{1}\right)$, and if this induced deformation satisfies a certain boundary condition, then $\left(W, w_{0}, \chi\right)$ is trivial (see $\S 1$, Main Theorem).

Our results may be regarded as an extension of those of A. Andreotti and E. Vesentini (see [2]) and our methods draw on theirs. In [2], one assumes that $\boldsymbol{M}$ is Hermitian, and considers the deformation of the complex structure on $M=M / \Gamma$ induced by a deformation $\left(W, w_{0}, \chi\right)$ of $\Gamma$ (assume again that $\Gamma$ contains no nontrivial elements of finite order). Roughly, their final result is that if this induced deformation satisfies a certain boundary condition (which they call rigidity at $\infty$ ), and if $\Gamma$ satisfies certain conditions, then $\left(W, w_{0}, \chi\right)$ is trivial. At present it is not clear how our boundary condition relates to that in [2], though it is not as stringent as rigidity at $\infty$ in the Riemannian sense, as defined in [5]. In two respects, our results are stronger than those in [2]. First, we need not assume $\boldsymbol{M}$ is Hermitian and second, we need make no restrictions on the lattice $\Gamma$, other than assuming $\Gamma$ contains no nontrivial elements of finite order. Moreover, in [2], the passage from cohomological results to deformation theoretic results is extremely complicated, involving delicate arguments from partial differential equations and several complex variables. However, in our framework this passage becomes relatively simple, and the fundamental idea is contained in Lemmas 5.6 and 5.23. below.

The text is organized into five sections. In $\S 1$ we show that when considering rigidity, it suffices to assume $W$ is an open interval; we then state our Main Theorem. In $\S \S 2$ and 3 , we reformulate in terms of locally constant vector bundles, some results in [1], concerning holomorphic vector bundles; this reformulation is direct, but is included for the sake of completeness. In $\S 4$ we reformulate some results in [2] and in [9], to obtain some global estimates (in the noncompact case) which, together with the results in $\S \S 2$ and 3, yield vanishing theorems for certain cohomology groups. Finally, we prove the Main Theorem in $\S 5$.

The author wishes to acknowledge stimulating discussions with S. Murakami and P. A. Griffiths. The author first proved Lemma 5.21 for the case when $G$ is simple and of even dimension. The argument for the case when $G$ is simple of odd dimension was shown to us by J. A. Wolf. Recently, G. D. Mostow found a somewhat simpler proof for Lemma 5.21, than the one presented here.

1. Statement of the Main Theorem. From now on we assume $G$ is admissible. Otherwise, we continue to use the notation already introduced in $\S 0$. If $M_{1}$ and $M_{2}$ are $C^{\infty}$ manifolds, and if $f: M_{1} \rightarrow M_{2}$ is a $C^{\infty}$ map, then $f_{*}$ will denote the

( ${ }^{1}$ It seems likely that one can reformulate the results in $\$ \S 3$ and 4 for $V$-manifolds, and thereby do away with the assumption that $\Gamma$ contains no elements of finite order; e.g. see [3]. 
tangent linear map to $f$. Now let $V$ be a vector space and let $S$ be a subset of End $V$. We then let $\mathscr{E}(S)$ denote the enveloping algebra of $S$.

Definition 1.1. A subset $H \subset G$ is called ample, in case $\mathscr{E}(\operatorname{Ad} H)=\mathscr{E}(\operatorname{Ad~} G)$.

Let $\zeta: G \rightarrow G / Z$ be the natural projection, where $Z$ is the center of $G$.

Lemma 1.2. Assume that $g_{i}$ is a sequence in $G$, that $H \subset G$ is ample, and that for all $h \in H$, the sequence $g_{i} h g_{i}^{-1}$ approaches $h$; then the sequence $\zeta\left(g_{i}\right)$ approaches the identity in $G / Z$.

Proof. From our assumption we have that for $h \in H$, the sequence Ad $g_{i} \operatorname{Ad} h \operatorname{Ad~} g_{i}^{-1}$ approaches $\operatorname{Ad} h$. Hence, since $H$ is ample, $\operatorname{Ad} g_{i} \operatorname{Ad} g$ Ad $g_{i}^{-1}$ approaches $\operatorname{Ad} g$ for all $g \in G$. Identifying the Lie algebra of Ad $G$ with $B$, we have that $\operatorname{Ad}_{i}(X)$ approaches $X$ for all $X \in \mathbb{B S}$. Hence $\operatorname{Ad} g_{i}$ approaches the identity

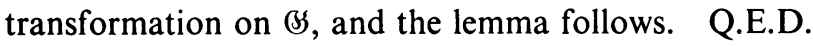

Corollary. Let $H$ be an ample subset of $G$; then the centralizer of $H$ in $G$ is $Z$.

Let $\theta: G \rightarrow \mathscr{R}$ be the map defined by

$$
\theta(g)(\gamma)=g \gamma g^{-1}, \quad g \in G, \quad \gamma \in \Gamma .
$$

From [4], $\Gamma$ is ample. Hence, by the Corollary to Lemma 1.2, $\theta$ induces a one-one map, $\theta^{\prime}: G / Z \rightarrow \mathscr{R}$. If $\Gamma_{1}$ is any ample subgroup of $G$, let $\mathscr{R}_{1}$ denote the space of all homomorphisms of $\Gamma_{1}$ into $G$, and define $\theta_{1}: G \rightarrow \mathscr{R}_{1}$ just as we defined $\theta$. Since $\Gamma_{1}$ is ample, we again have an induced one-one map, $\theta_{1}^{\prime}: G / Z \rightarrow \mathscr{R}_{1}$. If $\Gamma_{1}$ is finitely generated, with generators $\gamma_{1}, \ldots, \gamma_{s}$, we may identify $\mathscr{R}_{1}$ with a subset of $G^{s}=G \times \cdots \times G$ (s times). Namely, we identify $\sigma \in \mathscr{R}_{1}$ with the $s$-tuple $\left(\sigma\left(\gamma_{i}\right)\right)_{i}$. When convenient, we regard $\theta_{1}^{\prime}$ as a map from $G / Z$ to $G^{s}$, and we observe that as such, $\theta_{1}^{\prime}$ is a $C^{\infty}$ map.

LEMMA 1.4. Let $\Gamma_{1}$ be a finitely generated, ample subgroup; then $\theta_{1}^{\prime}$ is everywhere of maximal rank, and is a topological imbedding of $G / Z$ into $G^{s}$.

Proof. In order to show $\theta_{1}^{\prime}$ is of maximal rank, it suffices to show $\theta_{1}$ is of maximal rank. Let $g \cdot X$ be the left translate of $X \in \mathbb{B S}$ by $g \in G$, and assume $\left(\theta_{1}\right)_{*}(g \cdot X)=0$. It then follows that $\operatorname{Ad} \gamma(X)=X$, for all $\gamma \in \Gamma_{1}$. Since $\Gamma_{1}$ is ample, we must therefore have $X=0$, and thus $\theta_{1}^{\prime}$ is everywhere of maximal rank. The fact that $\theta_{1}^{\prime}$ is a topological imbedding follows from Lemma 1.2. Q.E.D.

Lemma 1.5. If $\left(W, w_{0}, \chi\right)$ is a deformation of $\Gamma$, and if $\chi(W) \subset \theta^{\prime}(G / Z)=\theta(G)$, then $\left(W, w_{0}, \chi\right)$ is trivial.

Proof. It suffices to find a $C^{\infty}$ map $c: W \rightarrow G$, such that $\theta \circ c=\chi$. We use the following observation of $\mathrm{H}$. C. Wang in [12]: $\Gamma$ contains a finitely generated, ample subgroup $\Gamma_{1}$. In fact, just choose $\gamma_{1}, \ldots, \gamma_{s} \in \Gamma$ so that the $\operatorname{Ad} \gamma_{i}(i=1, \ldots, s)$ 
generate $\mathscr{E}(\operatorname{Ad} \Gamma)$, and then let $\Gamma_{1}$ be the subgroup generated by the $\gamma_{i}$. Clearly $\Gamma_{1}$ is ample. We then have a commutative diagram

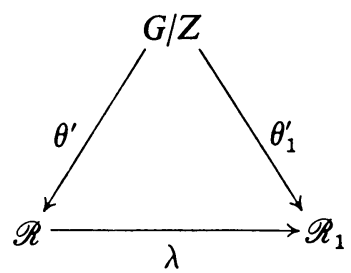

where $\lambda$ is a restriction map.

By our definition of smoothness for $\chi$, it follows that $\lambda \circ \chi$ is a $C^{\infty}$ map of $W$ into $G^{s}$. By assumption, $\chi(W) \subset$ Image $\theta^{\prime}$, so $\lambda \circ \chi(W) \subset$ Image $\theta_{1}^{\prime}$. Thus $\rho=\left(\theta_{1}^{\prime}\right)^{-1} \circ \lambda \circ \chi$ is a $C^{\infty}$ map from $W$ to $G / Z$, where we are here utilizing Lemma 1.4. Since $W$ is simply connected, we can find a $C^{\infty}$ map $c: W \rightarrow G$, such that $\zeta \circ c=\rho$. Moreover, $\theta \circ c=\left(\theta^{\prime} \circ \zeta\right) \circ c=\theta^{\prime} \circ(\zeta \circ c)=\theta^{\prime} \circ \rho=\theta^{\prime} \circ\left(\left(\theta_{1}^{\prime}\right)^{-1} \circ \lambda \circ \chi\right)$. On the image of $\chi$, which is contained in the image of $\theta^{\prime}$, we have $\left(\theta_{1}^{\prime}\right)^{-1} \circ \lambda=\left(\theta^{\prime}\right)^{-1}$. Therefore, $\theta \circ c=\chi$. Q.E.D.

For $w \in W$, let $\chi_{w}: \Gamma \rightarrow G$ be the monomorphism

$$
\chi_{w}(\gamma)=\chi(w)(\gamma), \quad \gamma \in \Gamma .
$$

We define an action of $\Gamma$ on $G \times W$ by

$$
(g, w) \gamma=\left(g \chi_{w}(\gamma), w\right), \quad g \in G, w \in W, \quad \gamma \in \Gamma .
$$

Let $\mathfrak{B}=(G \times W) / \Gamma$ denote the quotient space of $G \times W$ by this action of $\Gamma$, and let $\pi: G \times W \rightarrow \mathfrak{B}$ denote the natural projection. We make the regularity assumption

$$
\pi \text { is a covering map, and } \mathfrak{B} \text { is Hausdorff. }
$$

Thus $\mathfrak{B}$ inherits the structure of a $C^{\infty}$ manifold. We remark that (1.7) has been proved in the case when $G / \Gamma$ is compact (see [13] and [14]). Let $\operatorname{pr}_{2}: G \times W \rightarrow W$ denote the coordinate projection onto the second factor. Obviously $\mathrm{pr}_{2}$ commutes with the action of $\Gamma$ defined in (1.6), and hence induces a map $w: \mathfrak{B} \rightarrow W$, such that the diagram

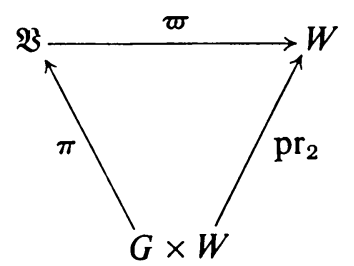

is commutative.

REMARK. In order to show that every lattice in $G$ is rigid, it suffices to show that for any open interval $I$, and for any lattice $\Gamma \subset G$, a deformation $(I, 0, \chi)$ of $\Gamma$ is locally trivial, in the sense that for some open interval $I^{\prime}$ with $0 \in I^{\prime} \subset I$, the deformation $\left(I^{\prime}, 0, \chi\right)$ is trivial. This remark follows from Lemma 1.5. Hence, from now 
on we will take $W$ to be an open interval containing zero, and we will shrink I (about 0) whenever convenient.

Let $t$ denote the parameter on $I$, and $\partial / \partial t$ the corresponding canonical vector field on $I$. The $\omega^{\lambda}$ induce forms on $G \times I$, and since these induced forms are invariant under the action (1.6) of $\Gamma$, the $\omega^{\lambda}$ also induce forms on $\mathfrak{B}$. In each case these induced forms will again be denoted by $\omega^{\lambda}$. From now on we make the convention that Greek indices $\lambda, \mu, \nu$ run from 1 to $n$. We then choose a basis of right-invariant vector fields $X_{\lambda}$ on $G$, such that with respect to the dual pairing with forms, we have $\left\langle X_{\lambda}, \omega^{\mu}\right\rangle=\delta_{\lambda}^{\mu}$. As with the $\omega^{\lambda}$, the $X_{\lambda}$ induce vector fields on $G \times I$ and on $\mathfrak{B}$, which we again denote by $X_{\lambda}$.

Using a partition of unity, we may define a $C^{\infty}$ vector field $Y$ on $\mathfrak{B}$, such that

$$
\varpi_{*}(Y)=\partial / \partial t
$$

Letting [ , ] denote the Poisson bracket, we have $\varpi_{*}\left(\left[X_{\lambda}, Y\right]\right)=\left[\varpi_{*} X_{\lambda}, \partial / \partial t\right]=0$, since $X_{\lambda}$ is vertical. Hence we may define $C^{\infty}$ functions $f_{\lambda}^{\mu}$, on $\mathfrak{B}$, by

$$
\left[X_{\lambda}, Y\right]=f_{\lambda}^{\mu} X_{\mu}
$$

where we use the Einstein summation convention for repeated indices. Let $f_{\lambda}^{t \mu}$ denote the restriction of $f_{\lambda}^{\mu}$ to $\varpi^{-1}(t)$ and let $\Gamma_{t}=\chi_{t}(\Gamma)$. Then we may identify $\varpi^{-1}(t)$ with $G / \Gamma_{t}$. We fix a Haar measure on $G$, and let $v_{t}$ denote the induced measure on $G / \Gamma_{t}$.

MAIN TheOREM. Let $\Gamma$ be a lattice in the admissible analytic group $G$; assume $\Gamma$ contains no nontrivial elements of finite order and let $(I, 0, \chi)$ be a deformation of $\Gamma$. Assume there exists a $C^{\infty}$ vector field $Y$ on $\mathfrak{B}$, such that (1.8) holds and such that for all $\lambda, \mu$

$$
\int_{G / \Gamma_{t}}\left|f_{\lambda}^{t \mu}\right|^{2} d v_{t}<\infty
$$

then $(I, 0, \chi)$ is trivial.

Corollary. If $(I, 0, \chi)$ is rigid at $\infty$ (see [5, Definition 5]), then it is trivial.

Using Lemma 1.5 and the subsequent remark, we see that the above Corollary strengthens the theorem in $[5$, p. 408] in that we need no longer assume $\Gamma$ is finitely generated. To get some feeling for the boundary condition (1.10), consider the case when $Y$ generates a one-parameter group of diffeomorphisms

$$
\theta_{t}: G / \Gamma \rightarrow G / \Gamma_{t} .
$$

If the $\theta_{t}$ are induced by right translations, the $f_{\lambda}^{\mu}$ are 0 , and conversely (see [14]). In general, the $f_{\lambda}^{\mu}$ may be regarded as a measure of how far the $\theta_{t}$ differ from being induced by right translations.

2. General cohomological results. Let $M$ be a connected, $C^{\infty}$, orientable, Riemannian manifold of dimension $\ell$. We fix an orientation of $M$, and let $d s^{2}$ denote the Riemannian metric on $M$. Relative to local coordinates $x^{1}, \ldots, x^{\ell}$, 
we have a representation of this metric

$$
d s^{2}=\sum_{i, j} g_{i j} d x^{i} d x^{j}
$$

Let $(M, M, \eta, \Gamma)$ denote a connected covering of $M$, where $\eta: M \rightarrow M$ is the covering map, and $\Gamma$ is the group of deck transformations of $\boldsymbol{M}$ (acting to the right). Let $\rho: \Gamma \rightarrow \operatorname{GL}\left(V_{\rho}\right)$ be a representation of $\Gamma$ in the finite-dimensional, real vector space $V_{\rho} .(M, M, \eta, \Gamma)$ may be regarded as a principal fiber bundle over $M$, with fiber $\Gamma$. With respect to $\rho$, we have the associated bundle $\left(M, E_{\rho}, \eta_{\rho}, V_{\rho}\right)$ with fiber $V_{\rho}$ and bundle projection $\eta_{\rho}: E_{\rho} \rightarrow M$. For short, we will denote this bundle by $E_{\rho}$. Let $m=\operatorname{dimension} V_{\rho}$ and fix a basis of $V_{\rho}$; we then have identifications of $V_{\rho}$ with $\boldsymbol{R}^{m}$ and of $\mathrm{GL}\left(V_{\rho}\right)$ with $\mathrm{GL}(m, \boldsymbol{R})$ (where $\boldsymbol{R}$ denotes the real numbers). Let $\boldsymbol{\rho}^{\prime}$ denote the contragredient representation of $\rho$; thus for $\gamma \in \Gamma, \rho^{\prime}(\gamma)={ }^{t} \rho(\gamma)^{-1}$, the inverse transpose of $\rho(\gamma)$.

Let $J$ be an index set, and let $\mathfrak{U}=\left\{U_{a}\right\}_{a \in J}$ be a covering of $M$ by open coordinate neighborhoods $U_{a}$, such that for each $U_{a}$ we have a diffeomorphism

$$
\Phi_{a}: U_{a} \times V_{\rho} \rightarrow \eta_{\rho}^{-1}\left(U_{a}\right),
$$

where $\eta_{\rho} \circ \Phi_{a}=\mathrm{pr}_{1}$, the coordinate projection of $U_{a} \times V_{\rho}$ onto the first factor. If $U_{a}$ meets $U_{b}$, we define

$$
e_{a b}: U_{a} \cap U_{b} \rightarrow \mathrm{GL}\left(V_{\rho}\right)
$$

by

$$
\Phi_{a}^{-1} \circ \Phi_{b}\left(x, v_{b}\right)=\left(x, e_{a b}(x) v_{b}\right), \quad x \in U_{a} \cap U_{b}, \quad v_{b} \in V_{\rho}
$$

We have

$$
\begin{aligned}
e_{a a} & =\text { identity, } \\
e_{a b} e_{b a} & =\text { identity }\left(\text { on } U_{a} \cap U_{b}\right), \\
e_{a b} e_{b c} & =e_{a c} \quad\left(\text { on } U_{a} \cap U_{b} \cap U_{c}\right) .
\end{aligned}
$$

Assume that for each $a \in J, h_{a}: U_{a} \rightarrow \mathrm{GL}(m, R)$ is a $C^{\infty}$ function, with values in the set of positive-definite, symmetric matrices. Assume, moreover, that on $U_{a} \cap U_{b}$

$$
h_{a}={ }^{t} e_{b a} h_{b} e_{b a} .
$$

We can use the $h_{a}$ to define a positive-definite inner product, $h$, on the fibers of $E_{\rho}$. Thus for $\boldsymbol{p}=\Phi_{a}\left(x, v_{a}\right), \boldsymbol{p}^{\prime}=\Phi_{a}\left(x, v_{a}^{\prime}\right)$, with $x \in U_{a}$, and $v_{a}, v_{a}^{\prime} \in \boldsymbol{R}^{m}$, we set $h\left(\boldsymbol{p}, \boldsymbol{p}^{\prime}\right)$ $={ }^{t} v_{a} h_{a}(x) v_{a}^{\prime}$. (2.2) then implies that $h$ is well defined.

Let $\mathscr{A}^{q}\left(E_{\rho}\right)$ denote the set of $C^{\infty}, E_{\rho}$-valued $q$-forms on $M$. Thus if $\varphi \in \mathscr{A}^{q}\left(E_{\rho}\right)$, then on $U_{a}, \varphi$ is given by a column vector

$$
\varphi_{a}=\left(\begin{array}{c}
\varphi_{a}^{1} \\
\vdots \\
\varphi_{a}^{m}
\end{array}\right),
$$


whose components are $C^{\infty}$ scalar valued $q$-forms on $U_{a}$. On $U_{a} \cap U_{b}$ we then have

$$
\varphi_{a}=e_{a b} \varphi_{b}
$$

We define

$$
d: \mathscr{A}^{q}\left(E_{\rho}\right) \rightarrow \mathscr{A}^{q+1}\left(E_{\rho}\right)
$$

by applying the ordinary exterior differentiation operator (which we also denote by d) componentwise. Since $E_{\rho}$ is a locally constant vector bundle, equation (2.3) implies $d$ is well defined.

Next, we define

$$
\hat{*}: \mathscr{A}^{q}\left(E_{\rho}\right) \rightarrow \mathscr{A}^{\ell-q}\left(E_{\rho^{\prime}}\right) .
$$

First, let $*$ denote the Hodge star operator defined on scalar forms by the Riemannian metric $d s^{2}$. Then, for $\varphi \in \mathscr{A}^{q}\left(E_{\rho}\right)$, set

$$
\hat{*} \varphi_{a}=h_{a}\left(* \varphi_{a}\right)
$$

where $*$ is here defined componentwise. A direct computation, using (2.1), (2.2), and (2.3), shows that $\hat{*}$ is well defined. Now define $\delta: \mathscr{A}^{q}\left(E_{\rho}\right) \rightarrow \mathscr{A}^{q-1}\left(E_{\rho}\right)$, by $\delta=(-1)^{q} \widehat{*}^{-1} d \hat{*}$. Finally, we define $\Delta: \mathscr{A}^{q}\left(E_{\rho}\right) \rightarrow \mathscr{A}^{q}\left(E_{\rho}\right)$, by $\Delta=d \delta+\delta d$.

Let $d X$ denote the volume element on $M$, with respect to $d s^{2}$ (recall that we have a fixed orientation on $M$ ). If $\phi, \psi \in \mathscr{A}^{q}\left(E_{\rho}\right)$, we can define a scalar $\ell$-form ${ }^{t} \varphi \wedge \hat{*} \psi$ on $M$, as follows: on $U_{a}$, we let ${ }^{t} \varphi \wedge \widehat{*} \psi$ equal ${ }^{t} \varphi_{a} \wedge \widehat{*} \psi_{a}$, where the latter is the matrix product of the row vector ${ }^{t} \varphi_{a}$ and the column vector $\hat{*} \psi_{a}$. A routine computation then shows that ${ }^{t} \varphi \wedge \widehat{*} \psi$ is well defined. We then define the $C^{\infty}$ function $\mathscr{Q}(\varphi, \psi)$ on $M$, by

$$
\mathcal{Q}(\varphi, \psi) d X={ }^{t} \varphi \wedge \widehat{*} \psi
$$

Whenever $\mathscr{2}(\varphi, \psi)$ is integrable (with respect to $d X)$, we define the inner product, $(\varphi, \psi)$, of $\varphi$ and $\psi$, by

$$
(\varphi, \psi)=\int_{M} \mathscr{2}(\varphi, \psi) d X
$$

The following is then a direct consequence of Stokes' theorem.

Proposition 2.5. Let $\varphi$ and $\psi$ be forms in $\mathscr{A}^{q}\left(E_{\rho}\right)$, such that support $\varphi \cap$ support $\psi$ is compact; then $(d \varphi, \psi)=(\varphi, \delta \psi)$ and $(\Delta \varphi, \psi)=(\varphi, \Delta \psi)=(d \varphi, d \psi)+(\delta \varphi, \delta \psi)$.

We conclude this section with a discussion of $W$-ellipticity and some of its consequences. Toward this end we first give some definitions and collect some elementary facts. Thus let $\mathscr{A}_{c}^{q}\left(E_{\rho}\right)$ denote the space of those forms in $\mathscr{A}^{q}\left(E_{\rho}\right)$ with compact support. We define a positive-definite, symmetric bilinear form, $a\left(\right.$, ), on $\mathscr{A}_{c}^{q}\left(E_{\rho}\right)$ by

$$
a(\varphi, \psi)=(\varphi, \psi)+(d \varphi, d \psi)+(\delta \varphi, \delta \psi), \quad \varphi, \psi \in \mathscr{A}_{c}^{q}\left(E_{\rho}\right)
$$


Let $\mathscr{L}^{q}=\mathscr{L}^{q}\left(E_{\rho}\right)$ and $\mathscr{W}^{q}=\mathscr{W}^{q}\left(E_{\rho}\right)$ denote the completions of $\mathscr{A}_{c}^{q}\left(E_{\rho}\right)$ with respect to the norms $\|\varphi\|=(\varphi, \varphi)^{1 / 2}$ and $N(\varphi)=a(\varphi, \varphi)^{1 / 2}$, respectively. We define $i: \mathscr{W}^{q} \rightarrow \mathscr{L}^{q}$ as follows: If $\varphi \in \mathscr{W}^{q}$, let $\varphi_{\nu}$ be a Cauchy sequence (with respect to $N(\quad)$ ) in $\mathscr{A}_{c}^{q}\left(E_{\rho}\right)$, representing $\varphi$. Then clearly $\varphi_{v}$ is also Cauchy with respect to $\|$. Hence $\varphi_{v}$ has a limit $i(\varphi)$ in $\mathscr{L}^{q}$, and it is obvious that $i(\varphi)$ is independent of the Cauchy sequence $\varphi_{\nu}$.

Proposition 2.6. $i$ is one-one.

Proof. Assume that $\varphi \in \mathscr{W}^{q}$ and that $i(\varphi)=0$. Then, by definition of $i$, we have a sequence $\varphi_{\nu}$ in $\mathscr{A}_{c}^{q}\left(E_{\rho}\right)$, representing $\varphi$, and such that

$$
\operatorname{Lim}_{\nu, \mu \rightarrow \infty} N\left(\varphi_{\nu}-\varphi_{\mu}\right)=\operatorname{Lim}_{\nu \rightarrow \infty}\left\|\varphi_{\nu}\right\|=0 .
$$

But then, for all $\psi \in \mathscr{A}_{c}^{q}\left(E_{\rho}\right)$, we have $\operatorname{Lim}_{v \rightarrow \infty}\left(\varphi_{v}, \psi\right)=0$; so in particular,

$$
\begin{gathered}
\operatorname{Lim}_{v \rightarrow \infty}\left(d \varphi_{v}, \psi^{\prime}\right)=\operatorname{Lim}_{v \rightarrow \infty}\left(\varphi_{v}, \delta \psi^{\prime}\right)=0, \\
\operatorname{Lim}_{v \rightarrow \infty}\left(\delta \varphi_{v}, \psi^{\prime \prime}\right)=\operatorname{Lim}_{v \rightarrow \infty}\left(\varphi_{v}, d \psi^{\prime \prime}\right)=0, \\
\psi^{\prime} \in \mathscr{A}_{c}^{q+1}\left(E_{\rho}\right), \quad \psi^{\prime \prime} \in \mathscr{A}_{c}^{q-1}\left(E_{\rho}\right) .
\end{gathered}
$$

Since $\varphi_{\nu}$ is Cauchy with respect to the norm $N(\quad)$, both $d \varphi_{\nu}$ and $\delta \varphi_{\nu}$ are Cauchy with respect to the norm \|\| . Let $d \varphi$ and $\delta \varphi$ denote the limits in $\mathscr{L}^{q+1}$ and $\mathscr{L}^{q-1}$, respectively, of the sequences $d \varphi_{\nu}$ and $\delta \varphi_{v}$, respectively. Then (2.7) implies $d \varphi$ and $\delta \varphi$ are zero. Hence, $\operatorname{Lim}_{v \rightarrow \infty}\left\|d \varphi_{v}\right\|=\operatorname{Lim}_{v \rightarrow \infty}\left\|\delta \varphi_{v}\right\|=\operatorname{Lim}_{v \rightarrow \infty}\left\|\varphi_{v}\right\|=0$, and therefore $\varphi=0$. Q.E.D.

REMARK. Using $i, \mathscr{W}^{q}$ may be identified with the subspace of all $\varphi \in \mathscr{L}^{q}$, such that there exists a sequence $\varphi_{v}$ in $\mathscr{A}_{c}^{q}\left(E_{\rho}\right)$, so that the sequences $\varphi_{v}, d \varphi_{v}$, and $\delta \varphi_{v}$ are Cauchy with respect to \|\| , and so that

$$
\operatorname{Lim}_{v \rightarrow \infty}\left\|\varphi-\varphi_{v}\right\|=0
$$

Let $d \varphi$ and $\delta \varphi$ denote the limits (with respect to $\|\quad\|$ ) of the sequences $d \varphi_{v}$ and $\delta \varphi_{v}$, respectively. Then Proposition 2.6 implies that $d \varphi$ and $\delta \varphi$ are independent of the sequence $\varphi_{v}$. Thus $d$ and $\delta$ have extensions to $\mathscr{W}^{q}$, in the sense of Friedrichs.

We define the Dirichlet inner product, $d\left(\right.$, ) on $\mathscr{W}^{q}$, by

$$
d(\varphi, \psi)=(d \varphi, d \psi)+(\delta \varphi, \delta \psi), \quad \varphi, \psi \in \mathscr{W}^{q} .
$$

We note that $d(\varphi, \varphi)^{1 / 2}$ defines a seminorm on $\mathscr{W}^{q}$. Recall that we have fixed a Riemannian metric $d s^{2}$ on $M$.

Definition 2.8. Let $h$ be a positive-definite inner product on the fibers of $E_{\rho}$; we then say that $E_{\rho}$ is $W^{q}$-elliptic relative to $h$, if there exists a constant $c>0$, so that for all $\varphi \in \mathscr{A}_{c}^{q}\left(E_{\rho}\right)$,

$$
c d(\varphi, \varphi) \geqq(\varphi, \varphi) .
$$


REMARK. Viewing $\mathscr{W}^{q}$ as the subspace of all $\varphi$ in $\mathscr{L}^{q}$, admitting $d \varphi$ and $\delta \varphi$ in the sense of Friedrichs, we note that (2.9) still has meaning on $\mathscr{W}^{q}$; then if $E_{\rho}$ is $W^{q}$-elliptic relative to $h,(2.9)$ still holds on $\mathscr{W}^{q}$. Thus, if $E_{\rho}$ is $W^{q}$-elliptic, $d(\varphi, \varphi)^{1 / 2}$ is a complete norm on $\mathscr{W}^{q}$, equivalent to $N(\varphi)$.

From now on, we will assume $h$ is fixed, and will speak of $W^{a}$-ellipticity without referring to $h$ explicitly.

THEOREM 2.10. If $E_{\rho}$ is $W^{q}$-elliptic, then for every $\alpha \in \mathscr{L}^{q}$, the equation

$$
\Delta x=\alpha
$$

has a unique weak solution $x \in \mathscr{W}^{q}$; that is, for any $u \in \mathscr{W}^{q}$ we have

$$
(d x, d u)+(\delta x, \delta u)=(\alpha, u) .
$$

Moreover, if $\alpha \in \mathscr{A}^{q}\left(E_{\rho}\right) \cap \mathscr{L}^{q}$, then $x \in \mathscr{A}^{q}\left(E_{\rho}\right) \cap \mathscr{W}^{q}$, and (2.11) holds in the classical sense.

Proof. Let $F$ be the linear functional on $\mathscr{W}^{q}$, defined by $F(u)=(\alpha, u), u \in \mathscr{W}^{q}$. Then from the Schwarz inequality and $W^{a}$-ellipticity, we have

$$
|F(u)| \leqq c^{1 / 2}\|\alpha\| d(u, u)^{1 / 2}, \quad u \in \mathscr{W}^{q} .
$$

Thus $F$ is continuous on $\mathscr{W}^{q}$, with respect to the complete norm $d(u, u)^{1 / 2}$ (see the remark following Definition 2.8). Hence, by the Riesz representation theorem, there exists a unique $x \in \mathscr{W}^{q}$, such that $F(u)=d(u, x), u \in \mathscr{W}^{q}$. But this proves the first assertion, while the last assertion follows from the fact that $\Delta$ is elliptic, and from the regularity theorem for such operators (see [7, pp. 268-269]). Q.E.D.

3. General cohomological results (continued). Fix a point $o \in M$ and for $p, q \in M$, let $\boldsymbol{d}(\boldsymbol{p}, \boldsymbol{q})$ denote the geodesic distance between $\boldsymbol{p}$ and $\boldsymbol{q}$. For $c>0$, let

$$
B(\boldsymbol{q}, c)=\{\boldsymbol{p} \in M \mid d(\boldsymbol{p}, \boldsymbol{q})<c\}, \quad B(c)=B(\boldsymbol{o}, c) .
$$

Given $\boldsymbol{q}$, we can assume $B(\boldsymbol{q}, c)$ is relatively compact, provided $c$ is sufficiently small. Moreover, if $d s^{2}$ is complete, then $B(q, c)$ is always relatively compact (see $[6$, p. 56, Theorem 10.3]).

A real-valued function $f$ on $\boldsymbol{R}^{\ell}$ is said to be locally Lipschitz, if for $\boldsymbol{p} \in \boldsymbol{R}^{\ell}$ we can find an open neighborhood $U$ of $\boldsymbol{p}$, and a constant $b>0$, so that for $\boldsymbol{p}_{1}, \boldsymbol{p}_{2}$ in $U,\left|f\left(\boldsymbol{p}_{1}\right)-f\left(\boldsymbol{p}_{2}\right)\right| \leqq b \sum_{i=1}^{\ell}\left|p_{1}^{i}-p_{2}^{i}\right|$, where $\boldsymbol{p}_{v}=\left(p_{v}^{1}, \ldots, p_{v}^{\ell}\right)$ for $\nu=1,2$. A real-valued function $f$ on $M$, is called locally Lipschitz, if for $p \in M$, we can find an open neighborhood $U$ of $\boldsymbol{p}$, and a diffeomorphism $g: \boldsymbol{R}^{\ell} \rightarrow U$, so that $f \circ g$ is locally Lipschitz on $\boldsymbol{R}^{\ell}$. It is easily seen that if $f$ is locally Lipschitz on $M$, and if $g: \boldsymbol{R}^{\ell} \rightarrow M$ is any $C^{\infty}$ map, then $f \circ g$ is locally Lipschitz on $\boldsymbol{R}^{\ell}$.

Lemma 3.1. $\rho(\boldsymbol{p})=\boldsymbol{d}(\boldsymbol{p}, \boldsymbol{o})$ is a locally Lipschitz $\left({ }^{2}\right)$ function. At points where the

$\left.{ }^{2}\right)$ In this section the symbol $\rho$ is used in two capacities: as a distance function and as a representation. We trust that this double usage will not cause any confusion. 
appropriate derivatives exist, we have in local coordinates

$$
\sum_{i, j=1}^{\ell} g^{i j}\left(\partial \rho / \partial x^{i}\right)\left(\partial \rho / \partial x^{j}\right) \leqq \ell .
$$

Proof. We prove the second assertion first. Thus, let $q$ be a point in $M$, and $U$ a normal coordinate neighborhood of $q$, with normal coordinates $\left(x^{1}, \ldots, x^{\ell}\right)$ on $U$. In particular, $\boldsymbol{q}$ has all coordinates zero. If $\boldsymbol{p} \in U$ has coordinates $\left(x^{1}, \ldots, x^{\ell}\right)$, then

$$
\boldsymbol{d}(\boldsymbol{p}, \boldsymbol{q})=\left(\left(x^{1}\right)^{2}+\cdots+\left(x^{\ell}\right)^{2}\right)^{1 / 2} .
$$

If the first derivatives of $\rho$ exist at $q$, we have at $\boldsymbol{q}$

$$
\begin{aligned}
\left|\partial \rho / \partial x^{i}\right| & =\left|\operatorname{Lim}_{y^{i} \rightarrow 0}\left(\rho\left(\boldsymbol{q}+\left(0, \ldots, \boldsymbol{y}^{i}, \ldots, 0\right)\right)-\rho(\boldsymbol{q})\right) / y^{i}\right| \\
& \leqq \operatorname{Lim}_{\boldsymbol{y}^{i} \rightarrow 0}\left|\boldsymbol{d}\left(\boldsymbol{q}+\left(0, \ldots, \boldsymbol{y}^{i}, \ldots, 0\right), \boldsymbol{q}\right)\right| /\left|\boldsymbol{y}^{i}\right|=1,
\end{aligned}
$$

where the last equality follows from (3.3). Since $g^{i j}=\delta_{i j}$ at $q,(3.2)$ now follows.

Now assume $c$ is small enough so that $B(q, c)$ is contained in a coordinate neighborhood $U$, with local coordinates $x^{1}, \ldots, x^{\ell}$. Let $T(M)$ denote the tangent bundle to $M$, and let $T(M) \mid B(q, c)$ denote the restriction of $T(M)$ to $B(q, c)$. Corresponding to the local coordinates $x^{1}, \ldots, x^{\ell}$ on $B(q, c)$, we have induced coordinates $\left(x^{1}, \ldots, x^{\ell} ; z^{1}, \ldots, z^{\ell}\right)$ on $T(M) \mid B(q, c)$, and we set $p=\left(x^{1}, \ldots, x^{\ell}\right)$, and $Z=\left(z^{1}, \ldots, z^{\ell}\right)$. If $c>0$ is small enough, we can find a neighborhood $\Omega$ of $B(q, c)$ in $T(M) \mid B(q, c)$, so that the map

$$
\Phi: \Omega \rightarrow B(q, c) \times B(q, c)
$$

defined by $\Phi(\boldsymbol{p}, Z)=\left(\exp _{\boldsymbol{p}}(Z), \boldsymbol{p}\right)$, is a diffeomorphism. Here $\operatorname{Exp}_{\boldsymbol{p}}$ denotes the exponential map with respect to $d s^{2}$, at $p$ (see $[8$, p. 166]). Let $|Z|$ denote the length of the tangent vector $Z$, with respect to $d s^{2}$. Passing to a smaller $c$, if necessary, we may assume that the matrix $\left(g_{i j}\right)$ has bounded eigenvalues on $B(q, c)$. Thus, there exists $e>0$, so that for $(p, Z) \in T(M) \mid B(q, c)$,

$$
|Z| \leqq e\left(\sum_{i=1}^{\ell}\left(z^{i}\right)^{2}\right)^{1 / 2} .
$$

If $p, p^{\prime} \in B(q, c)$ and if $p^{\prime}=\operatorname{Exp}_{p}(Z)$, then $d\left(p^{\prime}, p\right)=|Z|$. By (3.4), $|Z|$ is a locally Lipschitz function on $T(M) \mid B(q, c)$, and hence using $\Phi$, we see that $d\left(\boldsymbol{p}^{\prime}, \boldsymbol{p}\right)$ is a locally Lipschitz function on $B(q, c) \times B(q, c)$. Since

$$
\left|\rho(\boldsymbol{p})-\rho\left(\boldsymbol{p}^{\prime}\right)\right| \leqq \boldsymbol{d}\left(\boldsymbol{p}^{\prime}, \boldsymbol{p}\right)
$$

$\rho(\boldsymbol{p})$ is a locally Lipschitz function on $B(\boldsymbol{q}, c)$. Since $\boldsymbol{q} \in M$ was chosen arbitrarily, $\rho$ is a locally Lipschitz function on $M$. Q.E.D.

Let $V$ be an $\ell$-dimensional real vector space, let $F$ be an $m$-dimensional real vector space, and let $\bigwedge V$ denote the exterior algebra of $V$. We assume that $F$ and $V$ are equipped with positive-definite inner products. We then have induced 
inner products on $\bigwedge V$ and on $F \otimes \wedge V$. In each case we denote the induced inner product by $\langle$,$\rangle , and the corresponding norm by \mid$. For $u \in \wedge V$, let $\varepsilon(u): \wedge V \rightarrow \wedge V$ denote the corresponding left multiplication operator. We also let $\varepsilon(u)$ denote the operator on $F \otimes \wedge V$, obtained by tensoring the identity operator on $F$ with $\varepsilon(u)$ on $\bigwedge V$.

Lemma 3.5. If $u \in V, v \in F \otimes \wedge V$, then $|\varepsilon(u) v| \leqq|u||v|$.

Proof. Let $i(u): F \otimes \wedge V \rightarrow F \otimes \wedge V$ denote the adjoint of $\varepsilon(u)$ with respect to $\langle$,$\rangle . One then has$

$$
\varepsilon(u) i(u)+i(u) \varepsilon(u)=\langle u, u\rangle \mathrm{Id},
$$

where Id denotes the identity on $F \otimes \wedge V$. Thus

$$
\begin{aligned}
|\varepsilon(u) v|^{2} & =\langle\varepsilon(u) v, \varepsilon(u) v\rangle \\
& \leqq\langle\varepsilon(u) v, \varepsilon(u) v\rangle+\langle i(u) v, i(u) v\rangle \\
& =\langle(i(u) \varepsilon(u)+\varepsilon(u) i(u)) v, v\rangle=\langle u, u\rangle\langle v, v\rangle,
\end{aligned}
$$

where the last equality follows from (3.6). The lemma now follows by taking square roots. Q.E.D.

Corollary. Let $u$ be a scalar 1-form on $M$, and $v$ a q-form with values in $E_{\rho}$. We define $u \wedge v$ pointwise by $u \wedge v=\varepsilon(u) v$. At every point $p$ of $M$, we let $|u|$ denote the length of $u$ at $p$ with respect to $d s^{2}$. We then have at every point in $M$

$$
\mathscr{Q}(u \wedge v, u \wedge v) \leqq|u|^{2} \mathscr{Q}(v, v) .
$$

The following lemma follows from the fact that Stokes' theorem holds for locally Lipschitz differential forms. In particular, we remark that a locally Lipschitz form $\alpha$ is differentiable almost everywhere, so $d \alpha$ is defined almost everywhere.

LemMA 3.7. Let $\alpha$ be a locally Lipschitz q-form on $M$, with values in $E_{\rho}$; then for every $\varphi \in \mathscr{A}^{q}\left(E_{\rho}\right)$ such that support $\varphi \cap$ support $\alpha$ is compact, we have

$$
(d \varphi, d \alpha)+(\delta \varphi, \delta \alpha)=(\Delta \varphi, \alpha) .
$$

Let $\mu(t)$ be a $C^{\infty}$ function on $\boldsymbol{R}$ such that $0 \leqq \mu(t) \leqq 1$ and

$$
\begin{aligned}
\mu(t) & =1, \quad t \leqq 1, \\
& =0, \quad t \geqq 2 .
\end{aligned}
$$

Let $m=\sup |d \mu / d t|$, and let

$$
\omega(x)=\mu((\rho(x)+R-2 r) /(R-r)), \quad x \in M, \quad 0<r<R .
$$

Then (3.9) implies

$$
\begin{aligned}
0 & \leqq \omega(x) \leqq 1 \\
\omega(x) & =1, \quad x \in \overline{B(r)}, \\
& =0, \quad x \in M-B(R) .
\end{aligned}
$$


Also, a direct computation shows

$$
|d \omega / d \rho| \leqq m /(R-r) .
$$

For $\varphi \in \mathscr{A}^{q}\left(E_{\rho}\right),(3.10)$ implies that at every point in $M$, we have

$$
\mathscr{2}(\omega \varphi, \omega \varphi) \leqq \mathscr{Q}(\varphi, \varphi) .
$$

From (3.2) and (3.11), we have $|d \omega|^{2} \leqq \ell m^{2} /(R-r)^{2}$, wherever $d \omega$ is defined. From this and from the Corollary to Lemma 3.5, we have that wherever $d \omega$ is defined

$$
\mathscr{L}(d \omega \wedge \varphi, d \omega \wedge \varphi) \leqq \mathscr{Q}(\varphi, \varphi) \ell m^{2} /(R-r)^{2} .
$$

Set $\alpha=\omega^{2} \varphi$; so we have almost everywhere

$$
\begin{aligned}
& d \alpha=\omega^{2} d \varphi+2 \omega d \omega \wedge \varphi, \\
& \delta \alpha=\omega^{2} \delta \varphi+(-1)^{q} \hat{*}^{-1}(2 \omega d \omega \wedge \hat{*} \varphi) .
\end{aligned}
$$

For $c>0$, let $(\varphi, \varphi)_{B(c)}=\int_{B(c)} 2(\varphi, \varphi) d X$, and set $\|\varphi\|_{B(c)}=\left((\varphi, \varphi)_{B(c)}\right)^{1 / 2}$. We then have

Proposition 3.15. There exists a constant $a>0$, so that if $0<r<R$ and if $B(R)$ is relatively compact, then for $\sigma>0$ and $\varphi \in \mathscr{A}^{q}\left(E_{\rho}\right)$,

$$
\left(\|d \varphi\|_{B(r)}\right)^{2}+\left(\|\delta \varphi\|_{B(r)}\right)^{2} \leqq \sigma\left(\|\Delta \varphi\|_{B(R)}\right)^{2}+\left((1 / \sigma)+a /(R-r)^{2}\right)\left(\|\varphi\|_{B(R)}\right)^{2} .
$$

Proof. If we consider $(d \varphi, d \alpha)$ and $(\delta \varphi, \delta \alpha)$ and apply (3.14) and Lemma 3.7, we get

$$
\begin{aligned}
\left(\|\omega d \varphi\|_{B(R)}\right)^{2}+\left(\|\omega \delta \varphi\|_{B(R)}\right)^{2} \leqq & \left|\left(\Delta \varphi, \omega^{2} \varphi\right)_{B(R)}\right|+\left|(d \varphi, 2 \omega d \omega \wedge \varphi)_{B(R)}\right| \\
& +\left|\left(\delta \varphi, \hat{*}^{-1} 2 \omega d \omega \wedge \hat{*} \varphi\right)_{B(R)}\right| .
\end{aligned}
$$

Noting that for $\sigma>0,\left(\Delta \varphi, \omega^{2} \varphi\right)_{B(R)}=\left(\sigma \Delta \varphi,(1 / \sigma) \omega^{2} \varphi\right)_{B(R)}$, and applying the Schwarz inequality to each right-hand term in (3.17), we obtain

$$
\begin{aligned}
\left(\|\omega d \varphi\|_{B(R)}\right)^{2}+\left(\|\omega \delta \varphi\|_{B(R)}\right)^{2} \leqq & \sigma\left(\|\Delta \varphi\|_{B(R)}\right)^{2}+(1 / \sigma)\left(\left\|\omega^{2} \varphi\right\|_{B(R)}\right)^{2} \\
& +4\left(\|d \omega \wedge \varphi\|_{B(R)}\right)^{2}+4\left(\|d \omega \wedge \hat{*} \varphi\|_{B(R)}\right)^{2} .
\end{aligned}
$$

Applying (3.10) and (3.13) to the last three terms on the right of (3.18), we obtain $\left(\|\omega d \varphi\|_{B(R)}\right)^{2}+\left(\|\omega \delta \varphi\|_{B(R)}\right)^{2} \leqq \sigma\left(\|\Delta \varphi\|_{B(R)}\right)^{2}+\left((1 / \sigma)+8 \ell m^{2} /(R-r)^{2}\right)\left(\|\varphi\|_{B(R)}\right)^{2}$.

But, since $\omega=1$ on $B(r)$ and since $0 \leqq \omega \leqq 1$, we obtain (3.16), with $a=8 \mathrm{~lm}^{2}$. Q.E.D.

Proposition 3.19. If $d s^{2}$ is complete, then for all $\sigma>0$ and $\varphi \in \mathscr{A}^{q}\left(E_{\rho}\right)$, we have

$$
\|d \varphi\|^{2}+\|\delta \varphi\|^{2} \leqq \sigma\|\Delta \varphi\|^{2}+(1 / \sigma)\|\varphi\|^{2} .
$$

Proof. Since $d s^{2}$ is complete, $B(R)$ is relatively compact for all $R>0$. Thus, in (3.16), we can take $R=2 r$ and consider the limit of both sides as $r$ approaches infinity. The proposition now follows. Q.E.D. 
Proposition 3.20. Assume $d s^{2}$ is complete. If $\varphi \in \mathscr{L}^{q} \cap \mathscr{A}^{q}\left(E_{\rho}\right)$ and if $\Delta \varphi=0$, then $d \varphi=\delta \varphi=0$.

Proof. Just apply Proposition 3.19 and let $\sigma$ approach infinity. Q.E.D.

Proposition 3.21. If $x \in \mathscr{W}^{q} \cap \mathscr{A}^{q}\left(E_{\rho}\right)$, then $d x \in \mathscr{L}^{q+1}$, and $\delta x \in \mathscr{L}^{q-1}$,

Proof. There is a sequence $x_{v}$ in $\mathscr{A}_{c}^{q}\left(E_{\rho}\right)$, such that $\operatorname{Lim}_{v \rightarrow \infty}\left\|x_{v}-x\right\|=0$, and such that the sequences $d x_{v}$ and $\delta x_{v}$ are Cauchy with respect to $\|$. But then for $\psi \in \mathscr{A}_{c}^{q+1}\left(E_{\rho}\right)$

$$
\begin{aligned}
\operatorname{Lim}_{v \rightarrow \infty}\left(d x_{v}, \psi\right) & =\operatorname{Lim}_{v \rightarrow \infty}\left(x_{v}, \delta \psi\right) \\
& =(d x, \psi),
\end{aligned}
$$

where we are here utilizing Proposition 2.5. On the other hand, let $x^{\prime}$ be the limit in $\mathscr{L}^{q+1}$ of the Cauchy sequence $d x_{v}$. Thus, for $\psi \in \mathscr{A}_{c}^{q+1}\left(E_{\rho}\right)$

$$
\begin{aligned}
\left(x^{\prime}, \psi\right) & =\operatorname{Lim}_{v \rightarrow \infty}\left(d x_{v}, \psi\right) \\
& =\operatorname{Lim}_{v \rightarrow \infty}\left(x_{v}, \delta \psi\right)=(d x, \psi) .
\end{aligned}
$$

Hence $x^{\prime}=d x$, so $d x \in \mathscr{L}^{q+1}$. Similarly $\delta x \in \mathscr{L}^{q-1}$. Q.E.D.

THEOREM 3.22. Assume $d s^{2}$ is complete and $E_{\rho}$ is $W^{q}$-elliptic. If $\varphi \in \mathscr{L}^{q} \cap \mathscr{A}^{q}\left(E_{\rho}\right)$ and if $d \varphi=0$, then there exists $\psi \in \mathscr{L}^{q-1} \cap \mathscr{A}^{q-1}\left(E_{\rho}\right)$, such that $d \psi=\varphi$.

Proof. By $W^{q}$-ellipticity and Theorem 2.10 there exists $x \in \mathscr{W}^{q} \cap \mathscr{A}^{q}\left(E_{\rho}\right)$, such that $\Delta x=\varphi$. By assumption, $0=d \varphi=\Delta d x$, while from Proposition 3.21, we have $d x \in \mathscr{L}^{q+1}$. Hence, Proposition 3.20 implies that $\delta d x=0$. Thus $\varphi=\Delta x=d \delta x=d \psi$, where $\psi=\delta x$ is in $\mathscr{L}^{q-1} \cap \mathscr{A}^{q-1}\left(E_{\rho}\right)$. Q.E.D.

4. Cohomology on locally symmetric spaces. Our purpose in this section is to prove a vanishing theorem for "square integrable" cohomology (Theorem 4.17). We employ Theorem 3.22 and the methods in [9] (we refer to this paper for details omitted in this section). In fact, Theorem 4.17 is a reformulation of Theorem 7.1 in [9, Part I].

From now on, $G$ will denote an admissible analytic group and $\Gamma$ will denote a lattice in $G$. Recall that we have fixed a Cartan decomposition $\mathbb{S}=\mathfrak{H}+\mathfrak{P}$. We will let $r=\operatorname{dim} \mathfrak{P}$ and we will let Latin indices $i, j, k$ run from 1 to $r$, Greek indices $\alpha, \beta, \gamma$ run from $r+1$ to $n$, and Greek indices $\lambda, \mu, \nu$ run from 1 to $n$. Let (s) denote the dual space of $S$. The right-invariant one-forms $\omega^{\lambda}$ on $G$, determine corresponding element in $\mathbb{S}^{*}$, which we again denote by $\omega^{\lambda}$. Similarly, the right-invariant vector fields $X_{\lambda}$ determine a basis of $B S$, which is dual to the $\omega^{\lambda}$ (considered as elements of (S)*), and which we again denote by $X_{\lambda}$. We assume the $X_{\lambda}$ are chosen so that the $X_{i}$ are a basis for $\mathfrak{B}$, and the $X_{\alpha}$ are a basis for $\mathfrak{\pi}$. Moreover, if $B($, 
denotes the Killing form on (S), we assume the $X_{\lambda}$ are normalized so that

$$
\begin{aligned}
B\left(X_{\alpha}, X_{\beta}\right) & =-\delta_{\alpha \beta}, \\
B\left(X_{i}, X_{j}\right) & =\delta_{i j}, \\
B\left(X_{i}, X_{\alpha}\right) & =0 .
\end{aligned}
$$

We now define the positive-definite inner product, $(,)_{\mathrm{ad}}$, on \&S, by

$$
\left(X_{\lambda}, X_{\mu}\right)_{\mathrm{ad}}=\delta_{\lambda \mu} \text {. }
$$

In this section, we assume that $\Gamma$ contains no nontrivial elements of finite order; then the natural projection $\eta: \boldsymbol{M} \rightarrow \boldsymbol{M} / \Gamma$ is a covering map, and $M=\boldsymbol{M} / \Gamma$ is a $C^{\infty}$ manifold. (4.1) and the invariance of the Killing form, imply that $\sum_{i}\left(\omega^{i}\right)^{2}$ and ( , ) ad are $K$-invariant. Hence, in particular, $\sum_{i}\left(\omega^{i}\right)^{2}$ induces a $G$-invariant, and hence complete, Riemannian metric on $\boldsymbol{M}$. Hence $\sum_{i}\left(\omega^{i}\right)^{2}$ induces a complete Riemannian metric on $M$, and we take this metric to be the $d s^{2}$ of $\S \S 2$ and 3.

Let $\rho: G \rightarrow \mathrm{GL}\left(V_{\rho}\right)$ be a representation of $G$ in the $m$-dimensional, real vector space $V_{\rho}$; then $\rho$ induces a representation of $\Gamma$, which we again denote by $\rho$. We then have a locally constant vector bundle $\left(M, E_{\rho}, \eta_{\rho}, V_{\rho}\right)$ associated to the principal bundle $(M, M, \eta, \Gamma)$ by means of $\rho$. We let $\mathscr{E}_{\rho}$ denote the sheaf of germs of locally constant sections in $E_{\rho}$. Let $\mathscr{A}\left(E_{\rho}\right)$ be the direct sum of the $\mathscr{A}^{q}\left(E_{\rho}\right)$; then let $H^{q}\left(E_{\rho}\right)$ denote the $q$ th cohomology group of the complex $\left(\mathscr{A}\left(E_{\rho}\right), d\right)$. By the de Rham Theorem $H^{q}\left(E_{\rho}\right)$ is isomorphic to $H^{q}\left(M, \mathscr{E}_{\rho}\right)$, the $q$ th cohomology group of $M$, with coefficients in $\mathscr{E}_{\rho}$.

To use the machinery of harmonic analysis, introduced in $\$ \$ 2$ and 3 , we need only introduce a metric $h$ on the fibers of $E_{\rho}$. Toward this end, we first introduce the notion of an admissible, positive-definite inner product, $(,)_{\rho}$, on $V_{\rho}$. Thus, we say $(,)_{\rho}$ is admissible, in case the operators $\rho\left(X_{i}\right)$ and $\rho\left(X_{\alpha}\right)$ are symmetric and skew-symmetric, respectively, with respect to $(,)_{\rho}$. For each $\rho$, we fix an admissible, positive-definite inner product ( , ) $)_{\rho}$ (see [9, Part I, Lemma 3.1], for the proof that such inner products exist). One notes that $(,)_{a d}$, which we defined previously, is admissible, and for $\rho=$ ad we take this inner product to be our $(,)_{\rho}$. It follows from Proposition 3.1, in [9, Part I], that $(,)_{\rho}$ induces a positivedefinite inner product on the fibers of $E_{\rho}$, and we take $h$ to be this induced inner product.

Let $A^{q}(\Gamma, G, \rho)$ denote the space of all $V_{\rho}$-valued $q$-forms $\varphi$ on $G$, such that

$$
\varphi \circ R_{\gamma}=\rho(\gamma)^{-1} \quad \varphi, \gamma \in \Gamma,
$$

where $R_{\gamma}$ denotes the right translation on $G$, by $\gamma$. Let $A(\Gamma, G, \rho)$ denote the direct sum of the $A^{q}(\Gamma, G, \rho)$; then componentwise exterior differentiation induces a coboundary operator, $\hat{d}$, on $A(\Gamma, G, \rho)$. Let $\mathscr{A}(\Gamma, \boldsymbol{M}, \rho)$ denote the set of all $\varphi \in A(\Gamma, G, \rho)$, such that

$$
\varphi \circ L_{k}=\varphi, \quad k \in K, \quad i\left(X_{\alpha}\right) \varphi=0,
$$

where $L_{k}$ denotes the left translation on $G$, by $k$, and where $i\left(X_{\alpha}\right)$ denotes interior 
multiplication by the right-invariant vector field $X_{\alpha}$; then $\hat{d}$ leaves $\mathscr{A}(\Gamma, M, \rho)$ invariant. We let $d$ denote the restriction of $\hat{d}$ to $\mathscr{A}(\Gamma, M, \rho)$, and we let $\mathscr{A}^{q}(\Gamma, \boldsymbol{M}, \rho)=\mathscr{A}(\Gamma, \boldsymbol{M}, \rho) \cap A^{q}(\Gamma, G, \rho)$. From [9, pp. 368-369], we have that the complexes $(\mathscr{A}(\Gamma, \boldsymbol{M}, \rho), d)$ and $\left(\mathscr{A}\left(E_{\rho}\right), d\right)$ are isomorphic.

Given $\varphi \in A^{q}(\Gamma, G, \rho)$, we define the $V_{\rho}$-valued form $\varphi_{0}$ on $G$, by $\varphi_{0}(g)$ $=\rho(g) \varphi(g), g \in G$. Then, under the map $\varphi \rightarrow \varphi_{0}, A^{q}(\Gamma, G, \rho)$ corresponds to the space $A_{0}^{q}(\Gamma, G, \rho)$, of all $C^{\infty}, V_{\rho}$-valued forms, $\varphi$ on $G$, such that

$$
\varphi \circ R_{\gamma}=\varphi, \quad \gamma \in \Gamma ;
$$

and $\mathscr{A}^{q}(\Gamma, \boldsymbol{M}, \rho)$ corresponds to the subspace, $\mathscr{A}_{0}^{q}(\Gamma, \boldsymbol{M}, \rho)$, of all $\varphi$ in $A^{q}(\Gamma, G, \rho)$, such that

$$
\varphi \circ L_{k}=\rho(k) \varphi, \quad k \in K ; \quad i\left(X_{\alpha}\right) \varphi=0 .
$$

Under the correspondence $\varphi \rightarrow \varphi_{0}, \hat{d}$ and $d$ correspond to operators $\hat{d}^{0}$ on $A_{0}(\Gamma, G, \rho)$ and $d^{0}$ on $\mathscr{A}_{0}(\Gamma, M, \rho)$, respectively. Here $A_{0}(\Gamma, G, \rho)$ and $\mathscr{A}_{0}(\Gamma, M, \rho)$ denote the direct sums of the $A_{0}^{q}(\Gamma, G, \rho)$ and the $\mathscr{A}_{0}^{q}(\Gamma, \boldsymbol{M}, \rho)$, respectively. We have remarked that the complexes $(\mathscr{A}(\Gamma, \boldsymbol{M}, \rho), d)$ and $\left(\mathscr{A}\left(E_{\rho}\right), d\right)$ are isomorphic, and hence $\left(\mathscr{A}\left(E_{\rho}\right), d\right)$ is isomorphic to the complex $\left(\mathscr{A}_{0}(\Gamma, M, \rho), d^{0}\right)$. Our next goal will be to describe the operators $d^{0}, \delta^{0}$, and $\Delta^{0}$ on $\mathscr{A}_{0}(\Gamma, \boldsymbol{M}, \rho)$, corresponding to the operators, $d, \delta$, and $\Delta$, respectively, on $\mathscr{A}\left(E_{\rho}\right)$. We shall also describe $\hat{d}^{0}$ (see (4.20), below).

First, we can identify $A_{0}^{q}(\Gamma, G, \rho)$ with the space of all $C^{\infty}$ functions, $\varphi$, on $G$, with values in $V \rho \otimes \wedge^{q} \mathscr{S}^{*}$, such that

$$
\varphi(g \gamma)=\varphi(g), \quad g \in G, \quad \gamma \in \Gamma .
$$

We fix a basis $Y_{1}, \ldots, Y_{m}$ of $V_{\rho}$, which is orthonormal with respect to $(,)_{\rho}$. Then $\varphi \in A_{0}^{q}(\Gamma, G, \rho)$ has a representation $\varphi=f_{\lambda_{1} \cdots \lambda_{q}}^{s} Y_{s} \otimes \omega^{\lambda_{1}} \wedge \cdots \wedge \omega^{\lambda_{q}}$, where the $f_{\lambda_{1}}^{s} \cdots \lambda_{q}$ are $C^{\infty}$ functions on $G$ (alternating in $\lambda_{1}, \ldots, \lambda_{q}$ ), such that

$$
f_{\lambda_{1} \cdots \lambda_{q}}^{s}(g \gamma)=f_{\lambda_{1} \cdots \lambda_{q}}^{s}(g), \quad g \in G, \quad \gamma \in \Gamma .
$$

With regard to the above expression for $\varphi$, recall that we are using the Einstein summation convention.

We now define operators $\Theta^{\prime}\left(X_{\lambda}\right), \rho\left(X_{\lambda}\right), \Theta^{*}\left(X_{\lambda}\right), \varepsilon\left(\omega^{\lambda}\right)$, and $i\left(X_{\lambda}\right)$ on $A_{0}^{q}(\Gamma, G, \rho)$. First, let $i\left(X_{\lambda}\right): \wedge \mathscr{S}^{*} \rightarrow \wedge \mathscr{S}^{*}$ denote the adjoint of the left multiplication operator $\varepsilon\left(X_{\lambda}\right): \wedge \leftrightarrow H \rightarrow \wedge \mathscr{S}$. Then, for $\varphi \in A_{0}^{q}(\Gamma, G, \rho)$,

$$
\begin{aligned}
\Theta^{\prime}\left(X_{\lambda}\right) \varphi & =\left(X_{\lambda} f_{\lambda_{1}}^{s} \cdots \lambda_{q}\right) Y_{s} \otimes \omega^{\lambda_{1}} \wedge \cdots \wedge \omega^{\lambda_{q}} \\
\rho\left(X_{\lambda}\right) \varphi & =f_{\lambda_{1}}^{s} \cdots \lambda_{q}\left(\rho\left(X_{\lambda}\right) Y_{s}\right) \otimes \omega^{\lambda_{1}} \wedge \cdots \wedge \omega^{\lambda_{q}} \\
\Theta^{*}\left(X_{\lambda}\right) \varphi & =\sum_{j=1}^{q} f_{\lambda_{1}}^{s} \cdots \lambda_{q} Y_{s} \otimes \omega^{\lambda_{1}} \wedge \cdots \wedge \Theta^{*}\left(X_{\lambda}\right) \omega^{\lambda_{f}} \wedge \cdots \quad, \lambda_{q} \\
\varepsilon\left(\omega^{\lambda}\right) \varphi & =f_{\lambda_{1}}^{s} \cdots \lambda_{q} Y_{s} \otimes \omega^{\lambda} \wedge \omega^{\lambda_{1}} \wedge \cdots \wedge \omega^{\lambda_{q}} \\
i\left(X_{\lambda}\right) \varphi & =f_{\lambda_{1}}^{s} \cdots \lambda_{q} Y_{s} \otimes i\left(X_{\lambda}\right)\left(\omega^{\lambda_{1}} \wedge \cdots \wedge \omega^{\lambda_{q}}\right)
\end{aligned}
$$


where in the first equation $X_{\lambda} f_{\lambda_{1} \cdots \lambda_{q}}^{s}$ denotes the Lie derivative of $f_{\lambda_{1} \cdots \lambda_{q}}^{s}$ by the right-invariant vector field $X_{\lambda}$, and in the third equation, $\Theta *\left(X_{\lambda}\right) \omega^{\mu}$ is defined by $\left\langle\Theta *\left(X_{\lambda}\right) \omega^{\mu}, X_{v}\right\rangle=-\left\langle\omega^{\mu},\left[X_{\lambda}, X_{\nu}\right]\right\rangle$, with $\langle$,$\rangle here denoting the dual pairing$ between $\mathbb{S S}$ and $\mathbb{S S}^{*}$, and [, ] the Lie product on $\mathbb{S}$.

$\mathscr{A}_{0}^{q}(\Gamma, M, \rho)$ can now be described as the space of all $\varphi$ in $A_{0}^{q}(\Gamma, G, \rho)$, such that for all $\alpha$

$$
i\left(X_{\alpha}\right) \varphi=\Theta\left(X_{\alpha}\right) \varphi=0
$$

where

$$
\Theta\left(X_{\lambda}\right)=\rho\left(X_{\lambda}\right)+\Theta^{\prime}\left(X_{\lambda}\right)+\Theta^{*}\left(X_{\lambda}\right) .
$$

We can now describe the operators $d^{0}, \delta^{0}$, and $\Delta^{0}$ on $\mathscr{A}_{0}(\Gamma, M, \rho)$. Namely,

$$
d^{0}=D+d_{\rho}, \quad \delta^{0}=D_{*}+\delta_{\rho},
$$

where we set

$$
\begin{aligned}
d_{\rho}=\sum_{j} \rho\left(X_{j}\right) \varepsilon\left(\omega^{j}\right), & \delta_{\rho}=\sum_{j} \rho\left(X_{j}\right) i\left(X_{j}\right), \\
D=\sum_{j} \Theta^{\prime}\left(X_{j}\right) \varepsilon\left(\omega^{j}\right), & D_{*}=-\sum_{j} \Theta^{\prime}\left(X_{j}\right) i\left(X_{j}\right) .
\end{aligned}
$$

Of course, we have $\Delta^{0}=d^{0} \delta^{0}+\delta^{0} d^{0}$. However, it turns out that $\Delta^{0}$ has the following useful decomposition [10, Theorem 1]:

$$
\Delta^{0}=\Delta_{D}+\Delta_{\rho}
$$

where

$$
\Delta_{D}=D D_{*}+D_{*} D, \quad \Delta_{\rho}=d_{\rho} \delta_{\rho}+\delta_{\rho} d_{\rho} .
$$

In fact, (4.8) follows from a direct calculation, using (3.6) and (4.7). Another direct computation using (3.6), gives us

$$
\Delta_{\rho}=\sum_{i, j}\left[\rho\left(X_{i}\right), \rho\left(X_{j}\right)\right] \varepsilon\left(\omega^{i}\right) i\left(X_{j}\right)+\sum_{i} \rho\left(X_{i}\right)^{2} .
$$

By $\left(4.4^{\prime}\right), \varphi \in \mathscr{A}_{0}^{q}(\Gamma, M, \rho)$ induces a function on $G / \Gamma: \mathscr{A}_{c}^{q}\left(E_{\rho}\right)$ then corresponds to the space $\mathscr{A}_{0 c}^{q}(\Gamma, \boldsymbol{M}, \rho)$ of all $\varphi \in \mathscr{A}_{0}^{q}(\Gamma, M, \rho)$, such that $\varphi$ induces a function on $G / \Gamma$ with compact support. The positive-definite inner product ( , ) (see (2.4) for the definition), which is at least defined on $\mathscr{L}^{q} \cap \mathscr{A}^{q}\left(E_{\rho}\right)$, induces a positivedefinite inner product (still denoted by $($,$) ), which is at least defined on the$ subspace of $\mathscr{A}_{0}^{q}(\Gamma, \boldsymbol{M}, \rho)$ corresponding to $\mathscr{L}^{q} \cap \mathscr{A}^{q}\left(E_{\rho}\right)$.

In order to give an explicit description of this induced inner product, let $d v=\omega^{1} \wedge \cdots \wedge \omega^{n}$, where the $\omega^{\lambda}$ are here considered as one-forms on $G / \Gamma$; also, let

$$
\begin{aligned}
& \varphi=f_{i_{1} \cdots i_{q}}^{s} Y_{s} \otimes \omega^{i_{1}} \wedge \cdots \wedge \omega^{i_{q}}, \\
& \psi=g_{i_{1}}^{s} \cdots i_{q} Y_{s} \otimes \omega^{i_{1}} \wedge \cdots \wedge \omega^{i_{q}},
\end{aligned}
$$


be forms in $\mathscr{A}_{0}^{q}(\Gamma, M, \rho)$ (by virtue of (4.6), no $\alpha$ 's appear in these expressions for $\varphi$ and $\psi)$; then

$$
(\varphi, \psi)=e q ! \int_{G / \Gamma} \sum_{i_{1} \ldots i_{q}} f_{i_{1} \cdots i_{q}}^{s} g_{i_{1} \cdots i_{q}}^{s} d v,
$$

provided the right-hand integral is defined. Here, $e>0$ is a constant independent of $q, \varphi$, and $\psi$. Also, we note that $\mathscr{L}^{a} \cap \mathscr{A}^{q}\left(E_{\rho}\right)$ corresponds to the space of all $\varphi$ in $\mathscr{A}_{0}^{q}(\Gamma, \boldsymbol{M}, \rho)$, such that the integrals $\int_{G / \Gamma}\left(f_{i_{1} \ldots i_{q}}^{s}\right)^{2} d v$ are convergent for all $s, i_{1}, \ldots, i_{q}$.

For $\varphi \in A_{0 c}^{q}(\Gamma, M, \rho)$ and $\psi \in \mathscr{A}_{0 c}^{q+1}(\Gamma, M, \rho)$, we have

$$
\left(d^{0} \varphi, \psi\right)=\left(\varphi, \delta^{0} \psi\right), \quad\left(d_{\rho} \varphi, \psi\right)=\left(\varphi, \delta_{\rho} \psi\right), \quad(D \varphi, \psi)=\left(\varphi, D_{*} \psi\right),
$$

and hence, applying (4.9), we have for $\varphi \in \mathscr{A}_{0 c}^{q}(\Gamma, \boldsymbol{M}, \rho)$

$$
\left(\varphi, \Delta^{0} \varphi\right) \geqq 0, \quad\left(\varphi, \Delta_{D} \varphi\right) \geqq 0, \quad\left(\varphi, \Delta_{\rho} \varphi\right) \geqq 0 .
$$

Proposition 4.13. If there exists a constant $c>0$, such that for all

we have

$$
\varphi \in \mathscr{A}_{0 c}^{q}(\Gamma, M, \rho)
$$

$$
\left(\Delta_{\rho} \varphi, \varphi\right) \geqq c(\varphi, \varphi)
$$

then $E_{\rho}$ is $W^{q}$-elliptic.

Proof. From (4.8), $\left(\Delta^{0} \varphi, \varphi\right)=\left(\Delta_{\rho} \varphi, \varphi\right)+\left(\Delta_{D} \varphi, \varphi\right)$. However, (4.12) then implies $\left(\Delta^{0} \varphi, \varphi\right) \geqq\left(\Delta_{\rho} \varphi, \varphi\right)$, and combining this inequality with (4.13), we obtain the desired conclusion. Q.E.D.

We now restrict ourselves to the case when $q=1$. In this case, $\left(4.4^{\prime}\right)$ and (4.6) imply that $\varphi \in A_{0}^{1}(\Gamma, \boldsymbol{M}, \rho)$ may be regarded as a function on $G / \Gamma$, with values in Hom $\left(\mathfrak{B}, V_{\rho}\right)$. More explicitly, if $\varphi=f_{k}^{s} Y_{s} \otimes \omega^{k}$, then for $p \in G / \Gamma$, we identify $\varphi(p)$ with the element in $\operatorname{Hom}\left(\mathfrak{P}, V_{\rho}\right)$ defined by

$$
\varphi(\boldsymbol{p})\left(X_{i}\right)=f_{i}^{s}(\boldsymbol{p}) Y_{s}
$$

(where, using $\left(4.4^{\prime}\right)$, we identify the $f_{i}^{s}$ with functions on $G / \Gamma$ ).

We now define a positive-definite inner product $I($,$) on \operatorname{Hom}\left(\mathfrak{B}, V_{\rho}\right)$, by setting $I(\zeta, \xi)=\sum_{i}\left(\zeta\left(X_{i}\right), \xi\left(X_{i}\right)\right)_{\rho}, \zeta, \xi \in$ Hom $\left(\mathfrak{P}, V_{\rho}\right)$. Next, we define

$$
H: \operatorname{Hom}\left(\mathfrak{B}, V_{\rho}\right) \rightarrow \operatorname{Hom}\left(\mathfrak{B}, V_{\rho}\right)
$$

by

(4.15) $H(\zeta)(Y)=\sum_{k} \rho\left(X_{k}\right)^{2} \zeta(Y)+\sum_{k} \rho\left(\left[Y, X_{k}\right]\right) \zeta\left(X_{k}\right), \quad \zeta \in \operatorname{Hom}\left(\mathfrak{P}, V_{\rho}\right), \quad Y \in \mathfrak{P}$.

Using the fact that $(,)_{\rho}$ is admissible, one can show directly that $H$ is symmetric with respect to $I($,$) . Also, a direct computation using (4.10) and (4.14), shows$ that for $\varphi \in \mathscr{A}_{0 c}^{1}(\Gamma, M, \rho)$

$$
\left(\Delta_{\rho} \varphi, \varphi\right)=e \int_{G / \Gamma} I(H \varphi(\boldsymbol{p}), \varphi(\boldsymbol{p})) d v .
$$


THEOREM 4.17. Let $G$ be an admissible analytic group, $\Gamma \subset G$ a lattice containing no nontrivial elements of finite order, and $\rho: G \rightarrow \mathrm{GL}\left(V_{\rho}\right)$ a representation of $G$ in the finite-dimensional, real vector space $V_{\rho}$. Assume that the transformation $H: \operatorname{Hom}\left(\mathfrak{B}, V_{\rho}\right) \rightarrow \operatorname{Hom}\left(\mathfrak{B}, V_{\rho}\right)$, is positive-definite with respect to $I($, ). Then, if $\varphi \in \mathscr{A}_{0}^{1}(\Gamma, M, \rho)$ satisfies $(\varphi, \varphi)<\infty$ and $d^{0} \varphi=0$, there must then exist

$$
\psi \in \mathscr{A}_{0}^{0}(\Gamma, M, \rho)
$$

such that $d^{0} \psi=\varphi$.

Proof. Proposition 4.13, our assumption that $H$ is positive-definite with respect to $I($,$) , and (4.16) imply that E_{\rho}$ is $W^{1}$-elliptic. The assumption that $(\varphi, \varphi)<\infty$ implies that $\varphi$ corresponds to an element in $\mathscr{L}^{1} \cap \mathscr{A}^{1}\left(E_{\rho}\right)$, under our identification of $\mathscr{A}_{0}^{1}(\Gamma, M, \rho)$ with $\mathscr{A}^{1}\left(E_{\rho}\right)$. Thus the existence of $\psi$ follows from Theorem 3.22 and $W^{1}$-ellipticity. Q.E.D.

In fact, it is shown in [11], that most often $H$ is indeed positive-definite with respect to $I$. In particular, we have from [11] that $H$ is positive-definite for $\rho=a d$. Thus we have

Corollary. Let $G$ and $\Gamma$ be as in Theorem 4.17; then if $\varphi \in \mathscr{A}_{0}^{1}(\Gamma, M$, ad) satisfies $(\varphi, \varphi)<\infty$ and $d^{0} \varphi=0$, there must exist $\psi \in \mathscr{A}_{0}^{0}\left(\Gamma, M\right.$, ad) such that $d^{0} \psi=\varphi$.

We now give a brief discussion of the operator $\hat{d}^{0}$. First, we note that $A^{q}(\Gamma, G, \rho)$ may be identified with the space of all $C^{\infty}$ functions $\varphi$ on $G$, with values in $V_{\rho} \otimes \wedge^{q} \mathbb{S}^{*}$, and such that

$$
\varphi(g \gamma)=\left(\rho(\gamma)^{-1} \otimes \mathrm{Id}\right) \varphi(g),
$$

where $g \in G, \gamma \in \Gamma$, and Id denotes the identity map on $\bigwedge^{q}\left(\mathbb{S}^{*}\right)$. Using this identification, we define the operators $i\left(X_{\lambda}\right), \Theta^{\prime}\left(X_{\lambda}\right)$, and $\Theta^{*}\left(X_{\lambda}\right)$ on $A^{q}(\Gamma, G, \rho)$, just as we defined them on $A_{0}^{q}(\Gamma, G, \rho)$. We let $\tilde{\Theta}\left(X_{\lambda}\right)=\Theta^{\prime}\left(X_{\lambda}\right)+\Theta *\left(X_{\lambda}\right)$. Regarding $\varphi \in A^{q}(\Gamma, G, \rho)$ again as a $V_{\rho}$-valued $q$-form on $G$, and regarding $X_{\lambda}$ as a rightinvariant vector field on $G$, we note that $\tilde{\Theta}\left(X_{\lambda}\right)$ is simply the componentwise Lie derivative of $\varphi$, with respect to $X_{\lambda}$. We thus have the Cartan identity

$$
i\left(X_{\lambda}\right) \hat{d}+\hat{d i}\left(X_{\lambda}\right)=\Theta\left(X_{\lambda}\right) .
$$

Now a direct computation shows that under the correspondence $\varphi \rightarrow \varphi^{0}$, the operator $\Theta^{\prime}\left(X_{\lambda}\right)$ on $A^{q}(\Gamma, G, \rho)$ corresponds to $\rho\left(X_{\lambda}\right)+\Theta^{\prime}\left(X_{\lambda}\right)$ on $A_{0}^{q}(\Gamma, G, \rho)$. From this we obtain

$$
\hat{d}^{0}=\sum_{\lambda} \rho\left(X_{\lambda}\right) \varepsilon\left(\omega^{\lambda}\right)+\sum_{\lambda} \Theta^{\prime}\left(X_{\lambda}\right) \varepsilon\left(\omega^{\lambda}\right)+(1 / 2) \sum_{\lambda} \varepsilon\left(\omega^{\lambda}\right) \Theta *\left(X_{\lambda}\right),
$$

where we recall that $\hat{d}^{0}$ is the coboundary operator on $A_{0}(\Gamma, G, \rho)$, corresponding to $\hat{d}$ on $A(\Gamma, G, \rho)$, under the map $\varphi \rightarrow \varphi^{0}$. Also, we have from (4.19), that on $A_{0}(\Gamma, G, \rho)$

$$
i\left(X_{\lambda}\right) \hat{d}^{0}+\hat{d}^{0} i\left(X_{\lambda}\right)=\Theta\left(X_{\lambda}\right)
$$


We then have from (4.21)

Proposition 4.22. If $\varphi \in A_{0}^{q}(\Gamma, G, \rho)$ satisfies $i\left(X_{\alpha}\right) \varphi=0$ for all $\alpha$, and if $\hat{d}^{0} \varphi=0$, then $\Theta\left(X_{\alpha}\right) \varphi=0$ for all $\alpha$, and hence $\varphi \in \mathscr{A}_{0}^{q}(\Gamma, M, \rho)$.

We conclude this section with some formulae for the case $\rho=$ ad. Thus, if $\psi \in A_{0}^{0}\left(\Gamma, G\right.$, ad), then $\psi$ has an expression $\psi=h^{\lambda} X_{\lambda}$, where the $h^{\lambda}$ are $C^{\infty}$ functions on $G$ such that $h^{\lambda}(g \gamma)=h(g)$, for $g \in G$ and $\gamma \in \Gamma$. We note that $\psi$ may be regarded as a vector field on $G$. If $\varphi \in A_{0}^{1}\left(\Gamma, G\right.$, ad), then $\varphi$ has an expression $\varphi=f_{\mu}^{\lambda} X_{\lambda} \otimes \omega^{\mu}$, where the $f_{\mu}^{\lambda}$ are again $\Gamma$-invariant, $C^{\infty}$ functions on $G$, and we may regard $\sum_{\lambda} f_{\mu}^{\lambda} X_{\lambda}$ as a vector field on $G$. We then have

$$
\hat{d}^{0} \psi=\left[X_{\lambda}, \psi\right] \otimes \omega^{\lambda},
$$

where [ , ] here denotes the Poisson bracket of vector fields. Also, we have that $\hat{d}^{0} \varphi=0$, if and only if we have

$$
X_{v} f_{\mu}^{\lambda}-X_{\mu} f_{v}^{\lambda}=f_{\nu}^{\rho} c_{\mu \rho}^{\lambda}+f_{\rho}^{\lambda} c_{v \mu}^{\rho}-f_{\mu}^{\rho} c_{v \rho}^{\lambda},
$$

where we define the $c_{\lambda \mu}^{o}$ by $\left[X_{\lambda}, X_{\mu}\right]=c_{\lambda \mu}^{o} X_{\rho}$.

REMARK. By (4.4) we may regard the elements of $A_{0}(\Gamma, G, \rho)$, as differential forms on $G / \Gamma$. In particular, we may regard the $h^{\lambda}$ and $f_{\mu}^{\lambda}$, defined above, as functions on $G / \Gamma$, and we may then interpret (4.23) and (4.24) as holding on $G / \Gamma$.

5. Proof of the Main Theorem. Let $Z_{1}$ denote a central subgroup of $G$, and let $G^{\prime}=G / Z_{1}$. Let $p: G \rightarrow G^{\prime}$ denote the natural projection, and let $\Gamma^{\prime}=p(\Gamma)$. It then follows from Borel's density theorem (see [4]) that $\Gamma^{\prime}$ is a lattice in $G^{\prime}$. Moreover, a deformation $(I, 0, \chi)$ of $\Gamma$ induces a deformation $\left(I, 0, \chi^{\prime}\right)$ of $\Gamma^{\prime}$, with $p \circ \chi_{t}$ $=\chi_{t}^{\prime} \circ p$. In fact, this is a consequence of Borel's density theorem and the arguments in $[14, \S 16]$. We note that $(I, 0, \chi)$ is trivial if and only if $\left(I, 0, \chi^{\prime}\right)$ is, and it follows that we can assume without loss of generality, that $G$ has finite center, and hence that $K$ is compact.

We let $K$ act on $G \times I$ by

$$
k(g, t)=(k g, t), \quad k \in K, \quad g \in G, \quad t \in I .
$$

Since this action of $K$ obviously commutes with the action of $\Gamma$ defined by (1.6), we have an induced action of $K$ on $\mathfrak{B}$, which we will refer to as left translation.

Now assume $Y$ is a $C^{\infty}$ vector field on $\mathfrak{B}$ such that (1.8) and (1.10) hold. We first note that (1.10) is independent of which basis of right-invariant vector fields $X_{\lambda}$ we choose. Thus we assume the $X_{\lambda}$ are normalized so that (4.1) holds (where we here use the identification of (SS with right-invariant vector fields). For $k \in K$, we let $L_{k}$ denote the left translation by $k$ on $\mathfrak{B}$. We also let $L_{k}$ denote the induced maps on forms and vector fields on $\mathfrak{B}$. We then have

LEMMA 5.2. Let $Y$ be a $C^{\infty}$ vector field on $\mathfrak{B}$ satisfying (1.8) and (1.10); also, let 
$\tilde{Y}=\int_{K} L_{k} \cdot Y d k$. We define $C^{\infty}$ functions $\tilde{f}_{\lambda}^{\mu}$ on $\mathfrak{B}$ by $\left[X_{\lambda}, \tilde{Y}\right]=\tilde{f}_{\lambda}^{\mu} X_{\mu}$ and we let $\tilde{f}_{\lambda}^{t \mu}$ denote the restriction of $\tilde{f}_{\lambda}^{\mu}$ to $G / \Gamma_{t}$. We then have

$$
\int_{G / \Gamma_{t}}\left|\tilde{f}_{\lambda}^{t \mu}\right|^{2} d v_{t}<\infty
$$

for all $\lambda, \mu$, and for all $t \in I$.

Proof. For $g \in G$, we let (Ad $g)_{\mu}^{\lambda}$ denote the matrix coefficients of $\operatorname{Ad} g$, relative to the basis $X_{\lambda}$. We then have

$$
\tilde{f}_{\lambda}^{\mu}=\int_{K}\left(\operatorname{Ad~} k^{-1}\right)_{\lambda}^{\rho}(\operatorname{Ad} k)_{\sigma}^{\mu} L_{k} \cdot\left(f_{\rho}^{\sigma}\right) d k
$$

and (5.3) now follows from a direct calculation, using (1.10) and the compactness of $K$. Q.E.D.

Using Lemma 5.2, we see that the assumptions of the Main Theorem in $\S 1$ imply that there exists a $C^{\infty}$ vector field $Y$ on $\mathfrak{B}$, such that $L_{k} \cdot Y=Y$ for all $k \in K$, and such that (1.8) and (1.10) hold. From now on $Y$ will denote such a vector field. We let $\varphi^{t} \in A_{0}^{1}(\Gamma, G$, ad $)$ be defined by

$$
\varphi^{t}=f_{\mu}^{t \lambda} X_{\lambda} \otimes \omega^{\mu}
$$

From the Jacobi identity, we have

$$
\left[X_{v}\left[X_{\mu}, Y\right]\right]+\left[Y\left[X_{v}, X_{\mu}\right]\right]+\left[X_{\mu}\left[Y, X_{v}\right]\right]=0,
$$

and this implies directly, that the $f_{\mu}^{t \lambda}$ satisfy (4.24). Hence $\hat{d}^{0} \varphi^{t}=0$.

On the other hand $\left[X_{\alpha}, Y\right]=0$ for all $\alpha$, since $Y$ is invariant under left translations by elements of $K$. Thus the $f_{\alpha}^{t \lambda}$ are zero; that is, $i\left(X_{\alpha}\right) \varphi^{t}=0$ for all $\alpha$. Hence, by Proposition 4.22, $\varphi_{t} \in \mathscr{A}_{0}^{1}\left(\Gamma, M\right.$, ad). However, since $\varphi_{t}$ is a cocycle, we have from (1.10) and from the Corollary to Theorem 4.17, that there exists $\psi^{t} \in A_{0}^{0}(\Gamma, M$, ad $)$ such that $d^{0} \psi^{t}=\varphi^{t}$. From this, and (4.23) we have

$$
\left[X_{\lambda}, \psi^{t}\right]=f_{\lambda}^{t \mu} X_{\mu},
$$

where we can either regard $\psi^{t}$ as a vector field on $G / \Gamma_{t}$, or as a vector field on $G$.

For the moment, rather than considering the fibering $\varpi: \mathfrak{B} \rightarrow I$, we consider only the curve of homomorphisms $\chi_{t}$. Thus for $\gamma \in \Gamma$ and $s \in I$, let $\chi_{s}^{\prime}(\gamma)$ denote the tangent vector to the curve $\chi_{t}(\gamma)$ at $t=s$. We set $\gamma_{s}=\chi_{s}(\gamma)$ and let $f_{s}\left(\gamma_{s}\right)=\chi_{s}^{\prime}(\gamma) \gamma_{s}^{-1}$, the right translate of $\chi_{s}^{\prime}(\gamma)$ by $\gamma_{s}^{-1}$; then $f_{s}\left(\gamma_{s}\right) \in \mathcal{B S}$, and a direct computation shows

$$
f_{s}\left(\gamma_{s} \gamma_{s}^{\prime}\right)=f_{s}\left(\gamma_{s}\right)+\operatorname{Ad}\left(\gamma_{s}\right) f_{s}\left(\gamma_{s}^{\prime}\right), \quad \gamma, \gamma^{\prime} \in \Gamma
$$

Thus $f_{s}: \Gamma_{s} \rightarrow$ (S) is an Eilenberg-MacLane one-cocycle with respect to adjoint action. We will now use (5.4) to show

LEMMA 5.6. Under the hypotheses of the Main Theorem in $§ 1$, we have that for 
$s \in I$ there exists $b_{s} \in \mathbb{S}$, such that

$$
f_{s}\left(\gamma_{s}\right)=\operatorname{Ad} \gamma_{s}\left(b_{s}\right)-b_{s}, \quad \gamma_{s} \in \Gamma_{s}
$$

that is, $f_{s}$ is a coboundary.

Proof. We can assume without loss of generality, that $s=0$; then $\Gamma_{s}=\Gamma$, and $\gamma_{s}=\gamma$. We let $\pi_{0}: G \rightarrow G / \Gamma$ denote the natural projection, and for $\varepsilon>0$, we let $I_{\varepsilon}$ denote the open interval of radius $\varepsilon$ about 0 . Let $A^{\prime}$ be an open, relatively compact subset of $G / \Gamma$; we can then find $\varepsilon^{\prime}>0$ so that $I_{\varepsilon^{\prime}} \subset I$, and so that we have a $C^{\infty}$ diffeomorphism $\Lambda$, which maps $A^{\prime} \times I_{\varepsilon^{\prime}}$, onto an open subset of $\mathfrak{B}$, so that the diagram

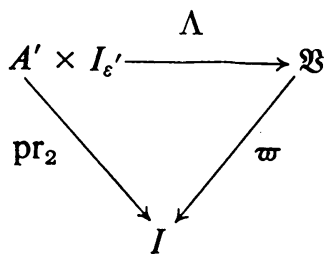

is commutative, and so that

$$
\Lambda(a, 0)=a, \quad a \in A^{\prime} .
$$

Now $\psi^{0}$ induces a vector field on $A^{\prime}$, and hence a vector field (still denoted by $\left.\psi^{0}\right)$ on $A^{\prime} \times I_{\varepsilon^{\prime}}$. We let $\hat{\psi}^{0}=\Lambda_{*}\left(\psi^{0}\right)$, so $\hat{\psi}^{0}$ is a vector field on $\Lambda\left(A^{\prime} \times I_{\varepsilon^{\prime}}\right)$. Let $U=\pi^{-1}\left(\Lambda\left(A^{\prime} \times I_{\varepsilon^{\prime}}\right)\right)$, and let $\Psi$ denote the lift of $\hat{\psi}^{0}$ to $U$ (so $\pi_{*} \Psi=\hat{\psi}^{0}$ ). Since (5.8) is commutative, we have that $\hat{\psi}^{0}$ and $\Psi$ are vertical in the sense that

$$
\varpi_{*} \hat{\psi}^{0}=0, \quad\left(\mathrm{pr}_{2}\right)_{*} \Psi=0 .
$$

We let $Y^{\prime}$ be the lift of $Y$ to $G \times I$, and then define the vector field $Y^{\prime \prime}$ on $U$ by $Y^{\prime \prime}=Y^{\prime}-\Psi$. We then have from (1.8) and (5.10), that

$$
\left(\mathrm{pr}_{2}\right)_{*} Y^{\prime \prime}=\left(\mathrm{pr}_{2}\right)_{*} Y^{\prime}=\partial / \partial t
$$

Hence on $U$ we have $Y^{\prime \prime}=Z+\partial / \partial t$, where $\left(\mathrm{pr}_{2}\right)_{*} Z=0$.

From (5.9) and the definition of $\Psi$, we have that $\Psi$ restricted to $\pi_{0}^{-1}\left(A^{\prime}\right) \times 0$ is equal to $\psi^{0}$. But then (5.4) implies

$$
\left[X_{\lambda}, Y^{\prime \prime}\right]=0, \text { on } \pi_{0}^{-1}\left(A^{\prime}\right) \times 0 \text {. }
$$

Since $Z$ is vertical, we can define a vector field $Z^{\prime}$ on $\pi_{0}^{-1}\left(A^{\prime}\right)$ by $Z^{\prime}(x)=Z(x, 0)$ for $x \in \pi_{0}^{-1}\left(A^{\prime}\right)$. Then (5.12) implies that $Z^{\prime}$ is left invariant; that is, we have

$$
Z^{\prime}(g x)=g Z^{\prime}(x), \quad g \in G, \quad x, g x \in \pi_{0}^{-1}\left(A^{\prime}\right) .
$$

Let $e$ denote the identity element of $G$, and set $e_{0}=\pi_{0}(e)$. Now assume that $A^{\prime}$ was chosen so as to contain $e_{0}$; then $\gamma \in \pi_{0}^{-1}\left(A^{\prime}\right)$ for all $\gamma \in \Gamma$. In order to prove the lemma, it will suffice to show

$$
f_{0}(\gamma)=\operatorname{Ad} \gamma\left(Z^{\prime}(e)\right)-Z^{\prime}(e), \quad \gamma \in \Gamma
$$

for we can then set $b_{0}=Z^{\prime}(e)$. 
To prove (5.14), we first choose an open, relatively compact subset $A$ of $A^{\prime}$, such that $e_{0} \in A \subset \bar{A} \subset A^{\prime}$. The vector field $Y-\hat{\psi}^{0}$ is defined on $\Lambda\left(A^{\prime} \times I_{\varepsilon^{\prime}}\right)$, and we will now consider the integral curves of this vector field. Thus, if we choose a sufficiently small positive number $\varepsilon$, we can assume that $0<\varepsilon<\varepsilon^{\prime}$, and that we have a $C^{\infty}$ map

$$
\kappa: A \times I_{\varepsilon} \rightarrow \Lambda\left(A^{\prime} \times I_{\varepsilon^{\prime}}\right)
$$

so that

$$
\kappa_{s}^{\prime}(x)=\left(Y-\hat{\psi}^{0}\right)\left(\kappa_{s}(x)\right), \quad x \in A, \quad s \in I_{\varepsilon},
$$

where we set $\kappa_{s}(x)=\kappa(x, s)$ and let $\kappa_{s}^{\prime}(x)$ denote the tangent to the curve $\kappa_{t}(x)$ at $t=s$. We let $\tilde{\kappa}: \pi_{0}^{-1}(A) \times I_{\varepsilon} \rightarrow \mathfrak{B}$ be the composition of $\kappa$ and of $\pi_{0} \times$ Id, where Id denotes the identity on $I_{\varepsilon}$.

By the covering homotopy theorem, there exists a map

$$
K: \pi_{0}^{-1}(A) \times I_{\varepsilon} \rightarrow G \times I,
$$

such that $K(x, 0)=(x, 0)$ for $x \in \pi_{0}^{-1}(A)$, and such that

$$
\pi(K(x, t))=\kappa\left(\pi_{0}(x), t\right), \quad x \in \pi_{0}^{-1}(A), \quad t \in I_{\varepsilon} .
$$

We observe that

$$
\operatorname{pr}_{2}\left(K_{t}(x)\right)=t, \quad x \in \pi_{0}^{-1}(A), \quad t \in I_{\varepsilon},
$$

where $K_{t}(x)=K(x, t)$. In fact, (5.17) follows from (5.11) and from

$$
K_{s}^{\prime}(x)=Y^{\prime \prime}\left(K_{s}(x)\right), \quad x \in \pi_{0}^{-1}(A), \quad s \in I_{\varepsilon},
$$

where $K_{s}^{\prime}(x)$ is the tangent to the curve $K_{t}(x)$ at $t=s$. On the other hand, (5.18) follows from (5.15).

From (5.17) we have $\pi\left(K_{t}(e) \gamma\right)=\pi\left(K_{t}(e)\right)$, where we use the right action of $\Gamma$ on $G \times I$ defined in (1.6). Then applying (5.16) to $\pi\left(K_{t}(e)\right)$ and to $\pi\left(K_{t}(\gamma)\right)$ and using the above equation, we get $\pi\left(K_{t}(e) \gamma\right)=\pi\left(K_{t}(\gamma)\right)$. But since $K_{0}(e) \gamma=K_{0}(\gamma)=\gamma$, we have by the unique lifting theorèm

$$
K_{t}(\gamma)=K_{t}(e) \gamma, \quad \gamma \in \Gamma, \quad t \in I_{\varepsilon} .
$$

Using (5.17), we define the one-parameter family of maps $K_{t}^{\#}: \pi_{0}^{-1}(A) \rightarrow G$ by $K_{t}(x)=\left(K_{t}^{\#}(x), t\right)$. From (5.19) we then have

$$
K_{t}^{\#}(\gamma)=K_{t}^{\#}(e) \chi_{t}(\gamma), \quad \gamma \in \Gamma, \quad t \in I_{\varepsilon} .
$$

From (5.18) we have that $Z^{\prime}(x)$ is the tangent vector to $K_{t}^{\#}(x)$ at $t=0$. Hence, differentiating both sides of (5.20) at $t=0$, we get $Z^{\prime}(\gamma)=Z^{\prime}(e) \gamma+\chi_{0}^{\prime}(\gamma)$. But (5.14), and hence the lemma, follows from this last equation by applying (5.13) to $Z^{\prime}(\gamma)$, and right-translating both sides of the equation by $\gamma^{-1}$. Q.E.D.

LEMMA 5.21. Given $s \in I$, we can find $\gamma^{1}, \ldots, \gamma^{\ell} \in \Gamma$, and real numbers $a_{1}, \ldots, a_{\ell}$, so 
that the linear transformation $\sum_{i=1}^{\ell} a_{i}\left(\mathrm{Id}-\mathrm{Ad} \gamma_{s}^{i}\right)$ is nonsingular (here Id denotes the identity on (S)).

Proof. Given real numbers $a_{1}, \ldots, a_{\ell}$, we define a polynomial function on $\mathrm{GL}($ (S) $) \times \cdots \times \mathrm{GL}(\mathcal{S})(\ell$ times $)$ by

$$
P\left(T_{1}, \ldots, T_{\ell}\right)=\operatorname{det}\left(\sum_{i=1}^{\ell} a_{i}\left(\mathrm{Id}-T_{i}\right)\right), \quad T_{1}, \ldots, T_{\ell} \in \mathrm{GL}(\mathrm{S}) .
$$

Now $G / \Gamma_{s}$ has finite invariant volume, so by Borel's density theorem (see [4]), the Zariski closure of $\operatorname{Ad} \Gamma_{s}$ contains $\operatorname{Ad} G$. Hence, if $P=0$ on $\operatorname{Ad} \Gamma_{s} \times \cdots \times \operatorname{Ad} \Gamma_{s}$ ( $l$ times), $P=0$ on Ad $G \times \cdots \times \operatorname{Ad} G$ ( $l$ times). Hence, the lemma will follow from

$$
\begin{aligned}
& \text { There are elements } g^{1}, \ldots, g^{\ell} \text { in } G \text { and real numbers } \\
& a_{1}, \ldots, a_{\ell} \text {, such that } \sum_{i=1}^{\ell} a_{i}\left(\operatorname{Id}-\operatorname{Ad} g^{i}\right) \text { is nonsingular. }
\end{aligned}
$$

However, to prove (5.22) it suffices to assume $G$ is simple. In that case the commuting algebra of $\operatorname{Ad} G$ is either $\boldsymbol{R}$ or $\boldsymbol{C}$. If $\operatorname{dim} G$ is even, then it follows that $\mathscr{E}(\operatorname{Ad} G)$, the enveloping algebra of $\operatorname{Ad} G$, must contain an element with no real eigenvalues. Hence there exist real numbers $a_{i}$, and elements $g^{i}$ in $G$, such that $\sum a_{i} \mathrm{Ad} g^{i}$ is nonsingular, and has no real eigenvalues. But then $\sum a_{i}\left(\operatorname{Id}-\operatorname{Ad} g^{i}\right)$ is nonsingular.

If $G$ is simple and $\operatorname{dim} G$ is odd (say $\operatorname{dim} G=2 k+1$ ), then the commuting algebra of $\operatorname{Ad} G$ is $R$, and $\mathscr{E}(\operatorname{Ad} G)$ is the full endomorphism algebra of $\mathscr{S}$. We fix a basis of BS, and we let

$$
\begin{aligned}
& S=\left(\begin{array}{rr}
0 & 1 \\
-1 & 0
\end{array}\right), \quad T_{1}=\left(\begin{array}{rrr}
0 & 0 & 0 \\
0 & 0 & 1 \\
0 & -1 & 0
\end{array}\right), \quad T_{2}=\left(\begin{array}{rrr}
1 & 1 & 0 \\
-1 & 0 & 0 \\
0 & 0 & 0
\end{array}\right) \\
& T_{1}=\left(\begin{array}{cccc}
T_{1} & & & 0 \\
& S & & \\
& & \cdot \bigcup_{S} & k-1 \text { times } \\
& & & \\
0 & & & S
\end{array}\right) \\
& \boldsymbol{T}_{2}=\left(\begin{array}{cccc}
T_{2} & & 0 \\
& S & & \\
& & \cdot & k-1 \text { times } \\
& & \multicolumn{2}{c}{S}
\end{array}\right)
\end{aligned}
$$


We then choose real numbers $b_{1}, \ldots, b_{q}, c_{1}, \ldots, c_{r}$, and $g^{1}, \ldots, g^{q}, h^{1}, \ldots, h^{r}$, in $G$, so that

$$
\sum_{i=1}^{q} b_{i} \operatorname{Ad} g^{i}=T_{1}, \quad \sum_{j=1}^{r} c_{j} \operatorname{Ad} h^{j}=T_{2} .
$$

If $\sum b_{i} \neq 0$ (resp. if $\sum c_{j} \neq 0$ ), then $\sum b_{i}\left(\operatorname{Id}-\operatorname{Ad} g^{i}\right)$ (resp. $\sum c_{j}\left(\operatorname{Id}-\operatorname{Ad} h^{j}\right)$ ) is nonsingular. Hence we may suppose $\sum b_{i}=\sum c_{j}=0$. But then $\sum b_{i}\left(\operatorname{Id}-\operatorname{Ad} g^{i}\right)$ $+\sum c_{j}\left(\mathrm{Id}-\operatorname{Ad} h^{j}\right)=\sum b_{i} \operatorname{Ad} g^{i}+\sum c_{j} \operatorname{Ad} h^{j}=T_{1}+T_{2}$, is nonsingular, so (5.22) (and hence the lemma) follows. Q.E.D.

LemMa 5.23. The $b_{t}(t \in I)$ in (5.7) are $C^{\infty}$ functions in $t$.

Proof. Fix $s \in I$, and then choose $\gamma^{i}$ and $a_{i}(i=1, \ldots, \ell)$ as in Lemma 5.21. Then $\sum_{i=1}^{\ell} a_{i}\left(\mathrm{Id}-\mathrm{Ad} \gamma_{t}^{i}\right)$ is nonsingular for $t$ in a sufficiently small interval $I^{\prime}$ containing $s$. But from (5.7), we have for $t \in I$

$$
\sum_{i=1}^{\ell} a_{i} f_{t}\left(\gamma_{t}^{i}\right)=\sum_{i=1}^{\ell} a_{i}\left(\operatorname{Ad} \gamma_{t}^{i}-\mathrm{Id}\right) b_{t}
$$

so that for $t \in I^{\prime}$, we have

$$
b_{t}=\left(\sum_{i=1}^{\ell} a_{i}\left(\mathrm{Ad} \gamma_{t}^{i}-\mathrm{Id}\right)\right)^{-1} \sum_{i=1}^{\ell} a_{i} f_{t}\left(\gamma_{t}^{i}\right) .
$$

But on $I^{\prime}$, the right-hand side of the above equation is a $C^{\infty}$ function of $t$. Hence $b_{t}$ is a $C^{\infty}$ function of $t$, on $I^{\prime}$. Since $s$ was chosen arbitrarily, $b_{t}$ is a $C^{\infty}$ function of $t$, on $I$. Q.E.D.

The lemma in $\left[8\right.$, p. 69] implies that there exists a $C^{1}$ curve $c_{t}$ in $G(t \in I)$ such that

$$
c_{s}^{\prime} c_{s}^{-1}=-b_{s}, \quad s \in I,
$$

where $c_{s}^{\prime}$ is the tangent to $c_{t}$ at $t=s$, and $c_{s}^{\prime} c_{s}^{-1}$ is the right translate of $c_{s}^{\prime}$ by $c_{s}^{-1}$; moreover, we can choose this curve $c_{t}$ so that $c_{0}=e$. An inspection of the proof of the Lemma in [8] mentioned above, shows that $c_{t}$ is in fact a $C^{\infty}$ curve. A direct computation, using (5.7) and (5.24), shows that the tangent vector to the curve $c_{t}^{-1} \chi_{t}(\gamma) c_{t}, \gamma \in \Gamma$, is zero for all $t \in I$. Therefore

$$
\chi_{t}(\gamma)=c_{t} \gamma c_{t}^{-1}, \quad \gamma \in \Gamma, \quad t \in I
$$

that is, $(I, 0, \chi)$ is a trivial deformation. The proof of the Main Theorem is now complete.

\section{BIBLIOGRAPHY}

1. A. Andreotti and E. Vesentini, Carleman estimates for the Laplace-Beltrami equation on complex manifolds, Inst. Hautes Études Sci. Publ. Math. 25 (1965), 81-130.

2. - On deformations of discontinuous groups, Acta. Math. 112 (1964), 249-298.

3. W. L. Baily, The decomposition theorem for V-manifolds, Amer. J. Math. 78 (1956), 862-888. 
4. A Borel, Density properties for certain subgroups of semisimple groups without compact components, Ann. of Math. (2) 72 (1960), 179-188.

5. H. Garland, On deformations of discrete groups in the noncompact case, Proc. Sympos. Pure Math. Vol. 9, pp. 405-412, Amer. Math. Soc., Providence, R. I., 1966.

6. S. Helgason, Differential geometry and symmetric spaces, Pure and Appl. Math. Vol. 12, Academic Press, New York, 1962.

7. L. Hörmander, Linear partial differential operators, Die Grundlehren der Mathematischen Wissenschaften Bd. 116, Academic Press, New York, 1963.

8. S. Kobayashi and K. Nomizu, Foundations of differential geometry, Tracts in Pure and Appl. Math. No. 15, Interscience, New York, 1963.

9. Y. Matsushima and S. Murakami, On vector bundle valued harmonic forms and automorphic forms on symmetric Riemannian manifolds, Ann. of Math. (2) 78 (1963), 365-416.

10. S. Murakami, Cohomologies of vector-valued forms on compact, locally symmetric Riemann manifolds, Proc. Sympos. Pure Math. Vol. 9, pp. 387-399, Amer. Math. Soc., Providence, R. I., 1966.

11. M. S. Raghunathan, On the first cohomology of discrete subgroups of semi-simple Lie groups, Amer. J. Math. 78 (1965), 103-139.

12. H. C. Wang, On a maximality property of discrete subgroups with fundamental domain of finite measure, Amer. J. Math. 89 (1967), 124-132.

13. A. Weil, On discrete subgroups of Lie groups, Ann. of Math. 72 (1960), 369-384.

14. - On discrete subgroups of Lie groups. II, Ann. of Math. 75 (1962), 578-602.

INSTITUTE FOR ADVANCED STUdy,

Princeton, New Jersey

YALE UNIVERSITY,

New Haven, Connecticut 